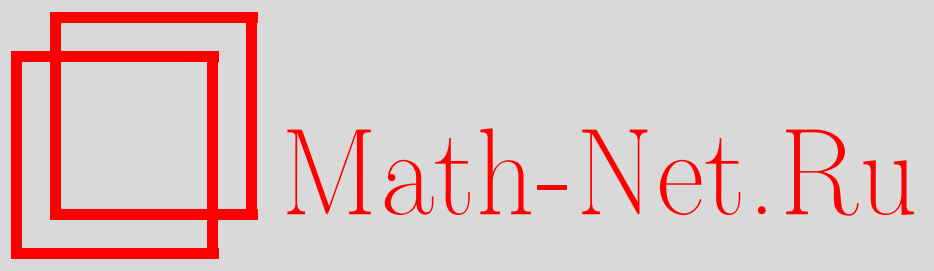

И. Т. Хабибуллин, М. В. Янгубаева, Формальная диагонализация дискретного оператора Лакса и законы сохранения и симметрии динамических систем, ТМФ, 2013, том 177, номер 3, 441-467

DOI: https://doi.org/10.4213/tmf8581

Использование Общероссийского математического портала Math-Net.Ru подразумевает, что вы прочитали и согласны с пользовательским соглашением http://www.mathnet.ru/rus/agreement

Параметры загрузки:

IP : 54.198 .55 .26

26 апреля 2023 г., 13:38:06

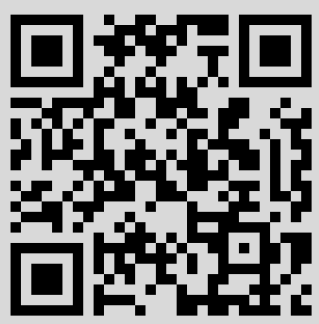




\title{
ФОРМАЛЬНАЯ ДИАГОНАЛИЗАЦИЯ ДИСКРЕТНОГО ОПЕРАТОРА ЛАКСА И ЗАКОНЫ СОХРАНЕНИЯ И СИММЕТРИИ ДИНАМИЧЕСКИХ СИСТЕМ
}

\begin{abstract}
Рассматривается задача построения формального асимптотического разложения по спектральному параметру для собственной функции дискретного линейного оператора. Предложен метод построения такого разложения, позволяющий находить законы сохранения для дискретных динамических систем, ассоциированных с заданным линейным оператором. В качестве иллюстративных примеров рассматриваются такие известные нелинейные модели, как дискретное потенциированное уравнение Котевега-де Фриза, дискретная версия нелинейного уравнения Шредингера с производной, одевающая цепочка Веселова-Шабата и другие. Описаны бесконечные серии законов сохранения для дискретной цепочки Тоды, соответствующей алгебре Ли $A_{1}^{(1)}$. Найдены новые примеры интегрируемых систем уравнений на квадратной решетке.
\end{abstract}

Ключевые слова: пара Лакса, асимптотические разложения, законы сохранения, симметрии, уравнения на квадратном графе, дискретное нелинейное уравнение Шредингера, метод одевания.

DOI: $10.4231 / \operatorname{tmf} 8581$

\section{1. ВВЕДЕНИЕ}

Асимптотическое по параметру поведение решений систем линейных дифференциальных уравнений хорошо исследовано (см., например, монографии [1], [2]). В последние несколько десятилетий интерес к этой тематике усилился благодаря приложениям в теории солитонов [3]-[7]. Например, метод обратной задачи рассеяния один из основных инструментов теории солитонов - полностью построен на асимптотическом анализе поведения собственных функций оператора Лакса. Известно также, что коэффициенты разложения собственной функции оператора Лакса по спектральному параметру определяют производящие функции законов сохранения для соответствующей динамической системы. Асимптотическая диагонализация пары Лакса позволила установить глубокие связи между интегрируемыми системами и бесконечномерными алгебрами Каца-Муди [8].

* Институт математики с вычислительным центром Уфимского научного центра РАН, Уфа, Россия. E-mail: habibullinismagil@gmail.com, marina.yangubaeva@mail.ru 
В настоящее время весьма активно исследуются дискретные динамические системы. Пары Лакса являются основным средством исследования нелинейных интегрируемых уравнений. В этой связи изучение свойств линейных систем вида

$$
y(n+1)=F(\mathbf{u}, \lambda) y(n)
$$

приобретает особую актуальность. Здесь $\mathbf{u}=\mathbf{u}(n)$ - функциональный параметр, являющийся векторнозначной функцией дискретного аргумента $n$. Насколько известно авторам, описание асимптотики системы (1.1) по спектральному параметру $\lambda$ остается мало исследованной проблемой даже в случае, когда $F(\mathbf{u}, \lambda)$ является полиномом от $\lambda$. Среди известных результатов можно выделить метод сведения уравнения (1.1) к нелинейному уравнению типа уравнения Риккати (см., например, монографии [4], [5]), который дает эффективный способ построения законов сохранения. К динамическим системам, содержащим дефекты, он применялся также в работах [9], [10]. В недавних работах [11], [12] такой способ был адаптирован к полностью дискретным моделям.

Следующий важный шаг в изучении дискретной линейной задачи был сделан А. В. Михайловым. Он предложил эффективную схему формальной диагонализации дискретных операторов, являющихся преобразованиями Дарбу для линейных дифференциальных уравнений. В работе [13] он заметил, что формальный ряд, диагонализующий дифференциальный оператор, диагонализует также все дискретные операторы, порожденные его преобразованиями Дарбу. Отметим, что такая схема позволяет строить законы сохранения для широкого класса уравнений в частных разностях и дифференциально-разностных уравнений [14].

В настоящей работе мы применяем известный в теории обыкновенных дифференциальных уравнений алгоритм формальной диагонализации (см., например, монографию [2]) непосредственно к дискретному линейному уравнению, без каких-либо дополнительных предположений о существовании связанных с ним линейных дифференциальных уравнений (см. также работу [15]). Эффективность такого подхода к построению формального асимптотического разложения собственных функций дискретного оператора иллюстрируется несколькими содержательными примерами.

Статья организована следующим образом. В разделе 2 мы доказываем предложение 1 , содержащеее объяснение метода диагонализации в дискретном случае. В разделе 3 обсуждается алгоритм построения законов сохранения по уже найденным диагональным представлениям. Разделы 4 и 5 посвящены обсуждению примеров. Алгоритм построения высших симметрий при помощи диагонализации обсуждается в разделе 6. В разделе 7 предлагаются новые примеры дискретных интегрируемых систем, в том числе дискретные версии "матричного" нелинейного уравнения Шредингера (НУШ) и "векторного" НУШ с производной.

\section{2. ФОРМАЛЬНАЯ ДИАГОНАЛИЗАЦИЯ ДИСКРЕТНОЙ ЛИНЕЙНОЙ СИСТЕМЫ}

Приведем сначала линейное уравнение (1.1) к некоторому специальному виду, удобному для построения асимптотического разложения. А именно, перепишем уравнение в следующем виде:

$$
y(n+1)=P(\mathbf{u}, \lambda) Z y(n),
$$


где $Z=\operatorname{diag}\left(\lambda^{\gamma_{1}}, \lambda^{\gamma_{2}}, \ldots, \lambda^{\gamma_{N}}\right)$ - диагональная матрица, показатели $\gamma_{i}$ - целые числа, причем все они попарно различны. Для простоты упорядочим показатели как

$$
\gamma_{1}>\gamma_{2}>\cdots>\gamma_{N}
$$

Мы предполагаем, что функция $P=P(\mathbf{u}(n), \lambda)$ зависит от дискретной переменной $n$ и параметра $\lambda$ и принимает свои значения в $\mathbb{C}^{N \times N}$. Кроме того, мы потребуем выполнения по крайней мере одного из следующих условий: для любого целого $n$ функция $P=P(\mathbf{u}(n), \lambda)$ является аналитической по $\lambda$ либо в некоторой окрестности точки $\lambda=\infty$, либо в некоторой окрестности точки $\lambda=0$. Некоторые дополнительные ограничения будут добавлены в предложении 1 ниже. Обсудим кратко, как привести дискретную систему к упомянутому выше специальному виду. Представление (2.1) возникло при изучении дискретной версии метода одевания по Захарову-Шабату.

Напомним суть метода одевания в наиболее простом его варианте. По заданной на контуре $|\lambda|=1$ матричнозначной функции $r=r(\lambda)$ построим новую функцию $r(n, \lambda)=Z^{n} r(\lambda) Z^{-n}$. Затем построим невырожденные матричнозначные функции $\phi(n, \lambda)$ и $\psi(n, \lambda)$, определенные на этом же контуре $|\lambda|=1$, которые допускают невырожденное аналитическое продолжение с контура в области $|\lambda|<1$ и $|\lambda|>1$ соответственно. Предполагается, что на самом контуре $|\lambda|=1$ выполняется равенство

$$
\phi(n, \lambda) r(n, \lambda)=\psi(n, \lambda) .
$$

В случае, когда $r=r(\lambda)$ достаточно близка к единичной матрице, задача (2.2) эффективно решается. Решение задачи единственно при дополнительном нормировочном условии. Например, можно предположить, что

$$
\psi(n, \infty)=1
$$

Теперь “одетый” потенциал легко вычисляется:

$$
F(\mathbf{u}, \lambda):=\phi(n+1, \lambda) Z \phi^{-1}(n, \lambda)=\psi(n+1, \lambda) Z \psi^{-1}(n, \lambda) .
$$

При этом, однако, возникает задача более явного (координатного) описания класса возможных значений потенциала $F(\mathbf{u}, \lambda)$. Например, в случае матриц размера $2 \times 2$ с матрицей $Z=\operatorname{diag}(\lambda, 1)$ и условием нормировки $(2.3)$ находим [16]

$$
F(\mathbf{u}, \lambda)=\left(\begin{array}{cc}
\lambda+u v & v \\
u & 1
\end{array}\right) .
$$

Здесь $\mathbf{u}=(u, v)-$ функциональный параметр.

В работе [15] показано, что потенциал $F(\mathbf{u}, \lambda)$ можно представить в виде

$$
F(\mathbf{u}, \lambda)=\alpha_{+}(\mathbf{u}, \lambda) Z \beta_{+}(\mathbf{u}, \lambda)=\alpha_{-}(\mathbf{u}, \lambda) Z \beta_{-}(\mathbf{u}, \lambda)
$$

где $\alpha_{ \pm}, \beta_{ \pm}$- треугольные матрицы, рациональным образом зависящие от параметра $\lambda$, которые имеют невырожденные пределы при $\lambda \rightarrow 0$ и при $\lambda^{-1} \rightarrow 0$. Следовательно, для таких потенциалов уравнение (1.1) можно при помощи линейных 
преобразований $z=\beta_{ \pm} y$ привести даже к двум различным уравнениям вида (2.1): мы имеем $z_{n+1}=D_{n}\left(\beta_{ \pm}\right) \alpha_{ \pm} Z z_{n}$. Вместо уравнения (1.1) удобно рассматривать связанное с ним уравнение с дискретным оператором $L=D_{n}^{-1} F$. Здесь $D_{n}$ - оператор сдвига, действующий по правилу $D_{n} f(n)=f(n+1)$. Рассуждения, приведенные выше, показывают, что при помощи сопряжения $L_{ \pm}=\beta_{ \pm} L \beta_{ \pm}^{-1}$ оператор $L$ можно привести к двум разным операторам специального вида:

$$
L_{ \pm}=D_{n}^{-1} P_{ \pm} Z, \quad P_{ \pm}=D_{n}\left(\beta_{ \pm}\right) \alpha_{ \pm}
$$

Для оператора с потенциалом (2.4) специальный вид найден в п. 4.1.

Следующее предложение, схема доказательства которого кратко обсуждалась в работе [15] одного из авторов, показывает, что форма (2.1) дискретного линейного уравнения является совершенно естественной с точки зрения асимптотических разложений. Отметим, что уравнение Лакса, как правило, задается в виде (1.1). Поэтому, чтобы воспользоваться предложением, нужно перейти от уравнения в форме (1.1) к специальной форме (2.1). Примеры, приведенные ниже, показывают, что искомый переход связан с разложением потенциала $F(\mathbf{u}, \lambda)$ в произведение треугольных сомножителей и с последующим калибровочным преобразованием.

ПРЕДЛОЖЕНИЕ 1. Рассмотрим дискретный оператор

$$
L=D_{n}^{-1} P(\mathbf{u}(n), \lambda) Z \text {. }
$$

Предположим, что для любого иелого числа $n$ функиия $P(\mathbf{u}(n), \lambda)$ является аналитической в некоторой окрестности точки $\lambda=\infty$,

$$
P(\mathbf{u}(n), \lambda)=P_{0}(n)+P_{1}(n) \lambda^{-1}+P_{2}(n) \lambda^{-2}+\cdots,
$$

и выполнено следующее условие регулярности: отличны от нуля все главные миноры матрици $P(\mathbf{u}(n), \infty)$,

$$
\operatorname{det}_{j} P(\mathbf{u}(n), \infty) \neq 0 \quad \text { для всех } j=1,2, \ldots, N \quad u \quad n \in(-\infty,+\infty) .
$$

Тогда существует формальный ряд

$$
T=\sum_{i \geqslant 0} T_{i} \lambda^{-i}
$$

такой, что оператор $L_{0}:=T^{-1} L T$ имеет вид $L_{0}=D_{n}^{-1} h Z$, где $h-\oint$ фомалъный ряд с диагональными коэфбициентами:

$$
h=h_{0}+h_{1} \lambda^{-1}+h_{2} \lambda^{-2}+\cdots .
$$

Ряд Т определяется с точностъю до умножения на формальный ряд с диагональными коэфбиииентами, его можно выбрать так, что все коэббициенты $T_{i} u h_{i}$ зависят от конечного, зависящего от $i$ набора переменных из $\{\mathbf{u}(k)\}_{k=-\infty,+\infty}$.

Если функиия $P=P(\mathbf{u}(n), \lambda)$ аналитична в окрестности точки $\lambda=0$,

$$
P(\mathbf{u}(n), \lambda)=P_{0}^{\prime}(n)+P_{1}^{\prime}(n) \lambda+P_{2}^{\prime}(n) \lambda^{2}+\cdots,
$$


и все главные миноры матрицы $P^{-1}(\mathbf{u}(n), 0)$ отличны от нуля,

$$
\underset{j}{\operatorname{det}} P^{-1}(\mathbf{u}(n), 0) \neq 0 \quad \text { для всех } \quad j=1,2, \ldots, N \quad u \quad n \in(-\infty,+\infty),
$$

то существует формальный ряд

$$
T^{\prime}=\sum_{i \geqslant 0} T_{i}^{\prime} \lambda^{i}
$$

такой, что оператор $L_{0}^{\prime}:=T^{\prime-1} L T^{\prime}$ имеет вид $L_{0}^{\prime}=D_{n}^{-1} h^{\prime} Z$, где $h^{\prime}$ - формалъный ряд с диагональными коэффициентами:

$$
h^{\prime}=h_{0}^{\prime}+h_{1}^{\prime} \lambda+h_{2}^{\prime} \lambda^{2}+\cdots .
$$

Ряд $T^{\prime}$ можно выбрать так, чтобы коэфбициенты $T_{i}^{\prime}$ и $h_{i}^{\prime}$ зависели от конечного, зависящего от $i$ набора переменных из $\{\mathbf{u}(k)\}_{k=-\infty,+\infty}$.

ДокАзАтЕЛьство. Докажем первую часть предложения, считая, что функция $P(\mathbf{u}(n), \lambda)$ аналитична в окрестности точки $\lambda=\infty$; вторая часть доказывается аналогично. Искомые объекты, ряды $T$ и $h$, определяются из уравнения

$$
D_{n}(T) h=P Z T Z^{-1}
$$

Ясно, что функция $\bar{T}(n, \lambda):=Z T(n, \lambda) Z^{-1}$ в силу условия

$$
\bar{T}(n, \lambda)=P^{-1}(\mathbf{u}(n), \lambda) D_{n}(T) h
$$

также аналитична на бесконечности. В результате имеем

$$
\bar{T}(\lambda)=\bar{T}_{0}+\bar{T}_{1} \lambda^{-1}+\bar{T}_{2} \lambda^{-2}+\cdots .
$$

Из очевидной формулы

$$
Z T Z^{-1}=\left(\begin{array}{cccc}
T_{1,1} & \lambda^{\gamma_{1,2}} T_{1,2} & \ldots & \lambda^{\gamma_{1, N}} T_{1, N} \\
\lambda^{\gamma_{2,1}} T_{2,1} & T_{2,2} & \ldots & \lambda^{\gamma_{2, N}} T_{2, N} \\
\vdots & \vdots & \ddots & \vdots \\
\lambda^{\gamma_{N, 1}} T_{N, 1} & \lambda^{\gamma_{N, 2}} T_{N, 2} & \ldots & T_{N, N}
\end{array}\right)
$$

где $\gamma_{i, j}=\gamma_{i}-\gamma_{j}$, следует, что элементы $\bar{T}_{i, j}$ матрицы $\bar{T}$ явным образом выражаются через элементы матрицы $T$ :

$$
\bar{T}_{i, j}=T_{i, j} \lambda^{\gamma_{i, j}} .
$$

Подставим в последнее равенство формальные ряды

$$
T_{i, j}(\lambda)=T_{0, i, j}+T_{1, i, j} \lambda^{-1}+\cdots, \quad \bar{T}_{i, j}(\lambda)=\bar{T}_{0, i, j}+\bar{T}_{1, i, j} \lambda^{-1}+\cdots,
$$

получим

$$
\bar{T}_{0, i, j}+\bar{T}_{1, i, j} \lambda^{-1}+\cdots=T_{0, i, j} \lambda^{\gamma_{i, j}}+T_{1, i, j} \lambda^{\gamma_{i, j}-1}+\cdots
$$


Из этого равенства легко можно получить связь между коэффициентами двух формальных рядов:

$$
\begin{aligned}
T_{p, i, j} & =\bar{T}_{p+\left|\gamma_{i, j}\right|, i, j} & \text { при } & i>j, \\
T_{p+\gamma_{i, j}, i, j} & =\bar{T}_{p, i, j} & \text { при } & i<j, \\
T_{p, i, i} & =\bar{T}_{p, i, i} . & &
\end{aligned}
$$

Заметим, что $\left|\gamma_{i, j}\right|=-\gamma_{i, j}$ при $i>j$ и $\left|\gamma_{i, j}\right|=\gamma_{i, j}$ при $i<j$. Если предположить, что $T_{\alpha, i, j}=\bar{T}_{\alpha, i, j}=0$ для $\alpha<0$, то равенствами $(2.9)-(2.11)$ можно пользоваться при всех целых значениях $p$.

Покажем, что матрицы $T_{0}$ и $\bar{T}_{0}$ являются соответственно нижне- и верхнетреугольными. Действительно, полагая $p=-\left|\gamma_{i, j}\right|$ в (2.9) и (2.10), находим, что $\bar{T}_{0, i, j}=0$ при $i>j$ и $T_{0, i, j}=0$ при $i<j$.

Теперь, сравнивая коэффициенты при нулевой степени $\lambda$ в уравнении (2.8), переписанном в виде

$$
D_{n}\left(T_{0}+\lambda^{-1} T_{1}+\cdots\right)\left(h_{0}+\lambda^{-1} h_{1}+\cdots\right)=\left(P_{0}+\lambda^{-1} P_{1}+\cdots\right)\left(\bar{T}_{0}+\lambda^{-1} \bar{T}_{1}+\cdots\right),
$$

получаем нелинейное уравнение относительно $T_{0}, h_{0}$ и $\bar{T}_{0}$ :

$$
D_{n}\left(T_{0}\right) h_{0}=P_{0} \bar{T}_{0}
$$

Это уравнение есть не что иное, как задача Гаусса о разложении матрицы $P_{0}$ в произведение трех матриц - нижнетреугольной $D_{n}\left(T_{0}\right)$, диагональной $h_{0}$ и верхнетреугольной $\bar{T}_{0}^{-1}$. Разрешимость этой задачи гарантируется условием регулярности (2.7) (см., например, монографию [17]). Допустим, что все диагональные элементы матрицы $T_{0}$ выбраны равными единице. Тогда очевидно, что диагональные элементы матриц $T_{0}$ и $\bar{T}_{0}$ совпадают, и решение задачи (2.13) единственно.

Далее мы полагаем, что диагональные элементы матрицы $T_{k}$ для всех $k>0$ равны нулю. Разложим каждую из матриц $T_{k}$ и $\bar{T}_{k}$ при $k>0$ в сумму нижнетреугольной и верхнетреугольной матриц с нулевой главной диагональю:

$$
T_{k}=T_{k \mathrm{~L}}+T_{k \mathrm{U}}, \quad \bar{T}_{k}=\bar{T}_{k \mathrm{~L}}+\bar{T}_{k \mathrm{U}} .
$$

Заметим, что матрицы $T_{1 \mathrm{U}}$ и $\bar{T}_{1 \mathrm{~L}}$ легко находятся. Действительно, если $\left|\gamma_{i, j}\right|=1$ для $i>j$, то из $(2.9)$ следует, что $\bar{T}_{1, i, j}=T_{0, i, j}$, т. е. этот элемент уже найден на предыдущем шаге. Если $\left|\gamma_{i, j}\right| \geqslant 2$, то из (2.9) следует, что $\bar{T}_{1, i, j}=T_{1-\left|\gamma_{i, j}\right|, i, j}=0$, поскольку индекс $\alpha=1-\left|\gamma_{i, j}\right|$ отрицательный. Следовательно, матрица $\bar{T}_{1 \mathrm{~L}}$ уже известна. Аналогично можно показать, что $T_{1 \mathrm{U}}$ также известна.

Для нахождения неизвестных $T_{1 \mathrm{~L}}$ и $\bar{T}_{1 \mathrm{U}}$ воспользуемся уравнением, полученным из (2.12) путем сравнения коэффициентов при $\lambda^{-1}$,

$$
D_{n}\left(T_{0}\right) h_{1}+D_{n}\left(T_{1}\right) h_{0}=P_{0} \bar{T}_{1}+P_{1} \bar{T}_{0},
$$

которое удобно переписать в виде

$$
h_{1} h_{0}^{-1}+D_{n}\left(T_{0}^{-1} T_{1 \mathrm{~L}}\right)-h_{0} \bar{T}_{0}^{-1} \bar{T}_{1 \mathrm{U}} h_{0}^{-1}=H_{1},
$$


где матрица $H_{1}=D_{n}\left(T_{0}^{-1}\right) P_{1} \bar{T}_{0} h_{0}^{-1}-D_{n}\left(T_{0}^{-1} T_{1 \mathrm{U}}\right)+h_{0} \bar{T}_{0}^{-1} \bar{T}_{1 \mathrm{~L}} h_{0}^{-1}$ содержит либо ранее найденные, либо заданные матрицы.

Теперь, чтобы найти неизвестные $h_{1}, D T_{1 \mathrm{~L}}$ и $\bar{T}_{1 \mathrm{U}}$, нужно разложить матрицу $H_{1}$ в сумму трех слагаемых - диагональной матрицы $h_{1} h_{0}^{-1}$, нижнетреугольной нильпотентной матрицы $D_{n}\left(T_{0}^{-1} T_{1 \mathrm{~L}}\right)$ и верхнетреугольной нильпотентной матрицы $h_{0} \bar{T}_{0}^{-1} \bar{T}_{1 \mathrm{U}} h_{0}^{-1}$. Продолжая этот процесс, можно найти все коэффициенты степенных рядов $T$ и $h$. Действительно, коэффициенты $T_{k}, h_{k}$ находятся из уравнений

$$
h_{k} h_{0}^{-1}+D_{n}\left(T_{0}^{-1} T_{k \mathrm{~L}}\right)-h_{0} \bar{T}_{0}^{-1} \bar{T}_{k \mathrm{U}} h_{0}^{-1}=H_{k},
$$

где член $H_{k}$ содержит слагаемые, найденные на предыдущем шаге. Для определения неизвестных $T_{k \mathrm{~L}}, \bar{T}_{k \mathrm{U}}, h_{k}$ достаточно разложить матрицу $H_{k}$ в сумму двух нильпотентных треугольных матриц и диагональной матрицы.

Допустим, что оператор $\widetilde{L}_{0}=\widetilde{T}^{-1} L \widetilde{T}$ имеет вид $\widetilde{L}_{0}=D^{-1} \tilde{h} Z$, и коэффициенты ряда $\tilde{h}$ также диагональны. Положим $S=T^{-1} \widetilde{T}$ и заметим, что выполняется равенство $\widetilde{L}_{0}=L_{0} S$, из которого легко следует, что $S-$ формальный ряд с диагональными элементами. Отсюда вытекает, что ряд $T$ определен с точностью до умножения справа на диагональный сомножитель. Предложение доказано.

\section{3. АЛГОРИТМ ПОСТРОЕНИЯ ЗАКОНОВ СОХРАНЕНИЯ}

Рассмотрим динамическую систему вида

$$
\epsilon\left(D_{m} D_{n} \mathbf{u}, D_{m} \mathbf{u}, D_{n} \mathbf{u}, \mathbf{u}\right)=0,
$$

где искомая функция $\mathbf{u}(n, m)=\left(u_{1}(n, m), u_{2}(n, m), \ldots, u_{N}(n, m)\right)$ зависит от двух целочисленных переменных $n, m$, а $D_{m}$ и $D_{n}$ - операторы сдвига по переменным $m$ и $n$ соответственно:

$$
D_{n} f(n, m)=f(n+1, m), \quad D_{m} f(n, m)=f(n, m+1) .
$$

Предположим, что система (3.1) допускает представление Лакса. Иначе говоря, уравнение (3.1) является условием совместности пары линейных уравнений вида

$$
y(n+1, m)=P([\mathbf{u}], \lambda) Z y(n, m), \quad y(n, m+1)=R([\mathbf{u}], \lambda) y(n, m) .
$$

Заметим, что первое из двух уравнений записано в форме (2.1). Здесь запись вида $P=P([\mathbf{u}], \lambda), R=R([\mathbf{u}], \lambda)$ указывает на то, что функции $P$ и $R$ зависят от переменной $\mathbf{u}$ и конечного числа ее сдвигов $D_{n}^{k} \mathbf{u}, D_{m}^{k} \mathbf{u}$. Легко проверить, что условие совместности системы уравнений (3.2) записывается как

$$
D_{m}(P) Z R=D_{n}(R) P Z
$$

Введем в рассмотрение операторы $L=D_{n}^{-1} P Z$ и $M=D_{m}^{-1} R$. Тогда уравнение $(3.3)$ принимает вид $[L, M]=0$. Допустим, что функция $P([\mathbf{u}], \lambda)$ удовлетворяет всем условиям предложения 1 (она аналитична в окрестности точки $\lambda=\infty$ для всех значений переменной $\mathbf{u}$, лежащих в некоторой области, а главные миноры матрицы $P([\mathbf{u}], \infty)$ отличны от нуля в этой области). Допустим далее, что функция 
$R([\mathbf{u}], \lambda)$ мероморфна в окрестности точки $\lambda=\infty$, когда $\mathbf{u}$ принимает свои значения в упомянутой области. Тогда операторы $L$ и $M$ приводятся к диагональному виду при помощи одного и того же преобразования. Теперь, следуя схеме, предложенной в работе [8] (см. также [13] и обзор [18]), можно найти производящую функцию законов сохранения динамической системы (3.1). Действительно, в соответствии с предложением 1 имеем $L_{0}=T^{-1} L T$, где оператор $L_{0}=D_{n}^{-1} h Z^{n}$ диагональный. Положим $M_{0}:=T^{-1} M T$. По построению и в силу предположения, сделанного выше, коэффициент $S$ в формуле $M_{0}=D_{m}^{-1} S$ является формальным рядом вида $S=\lambda^{k} \sum_{i=0}^{\infty} S_{i} \lambda^{-i}$.

ПреДЛОЖЕНИЕ 2. Коэффициенты $S_{i}$ ряда $S$ являются диагоналъными матрииами.

ДокАзАТЕЛЬСтво. Из уравнения $\left[L_{0}, M_{0}\right]=0$ следует, что

$$
D_{n}(S) h=D_{m}(h) Z S Z^{-1} \text {. }
$$

Положим $\bar{S}=Z S Z^{-1}=\bar{S}_{0}+\bar{S}_{1} \lambda^{-1}+\bar{S}_{2} \lambda^{-2}+\cdots$. Ясно, что ряд $\bar{S}$ не может содержать положительных степеней $\lambda$, поскольку $\bar{S}=D_{m}\left(h^{-1}\right) D_{n}(S) h$, причем $\operatorname{det} h_{0} \neq 0$ в силу условий регулярности (2.7) предложения 1. Легко видеть, что элементы матриц $S(\lambda)$ и $\bar{S}(\lambda)$ связаны соотношениями $\bar{S}_{i, j}(\lambda)=S_{i, j}(\lambda) \lambda^{\gamma_{i, j}}$, где введены обозначения

$$
S_{i, j}(\lambda)=S_{0, i, j}+S_{1, i, j} \lambda^{-1}+\cdots, \quad \bar{S}_{i, j}(\lambda)=\bar{S}_{0, i, j}+\bar{S}_{1, i, j} \lambda^{-1}+\cdots .
$$

В результате имеем $\bar{S}_{i, j}(\lambda)=S_{0, i, j} \lambda^{\gamma_{i, j}}+S_{1, i, j} \lambda^{\gamma_{i, j}-1}+\cdots$. Сравнивая коэффициенты двух различных представлений для $\bar{S}_{i, j}(\lambda)$, получаем формулы, связывающие коэффициенты рядов $S(\lambda)$ и $\bar{S}(\lambda)$ :

$$
\begin{array}{rlrl}
S_{p, i, j} & =\bar{S}_{p+\left|\gamma_{i, j}\right|, i, j} & \text { при } \quad i>j, \\
S_{p+\gamma_{i, j}, i, j} & =\bar{S}_{p, i, j} & \text { при } & i<j, \\
S_{p, i, i} & =\bar{S}_{p, i, i} . & &
\end{array}
$$

Полагая в (3.5) и (3.6) $p=-\left|\gamma_{i, j}\right|$, получаем $\bar{S}_{0, i, j}=S_{-\left|\gamma_{i, j}\right|, i, j}=0$ при $i>j$ и $S_{0, i, j}=\bar{S}_{-\gamma_{i, j}, i, j}=0$ при $i<j$, так как элементы $S_{\alpha, i, j}$ и $\bar{S}_{\alpha, i, j}$ равны нулю, если индекс $\alpha$ отрицателен. Отсюда имеем, что матрицы $S_{0}$ и $\bar{S}_{0}$ являются нижнеи верхнетреугольными соответственно. Подставим в (3.4) вместо $S$ и $h$ формальные ряды

$$
D_{n}\left(\sum_{p=0}^{\infty} S_{p} \lambda^{-p}\right)\left(\sum_{q=0}^{\infty} h_{q} \lambda^{-q}\right)=D_{m}\left(\sum_{q=0}^{\infty} h_{q} \lambda^{-q}\right)\left(\sum_{p=0}^{\infty} \bar{S}_{p} \lambda^{-p}\right)
$$

и сравним коэффициенты при $\lambda^{0}$ :

$$
D_{n}\left(S_{0}\right) h_{0}=D_{m}\left(h_{0}\right) \bar{S}_{0} .
$$

Отсюда в силу невырожденности диагональных матриц $h_{0}, D_{m}\left(h_{0}\right)$ и треугольного вида матриц $S_{0}, \bar{S}_{0}$ имеем, что $S_{0}=\bar{S}_{0}$ есть диагональная матрица. 
Рассмотрим следующие коэффициенты $S_{1}, \bar{S}_{1}$. Для тех элементов $\bar{S}_{1, i, j}$ матрицы $\bar{S}_{1}$, для которых при $i>j$ выполнено условие $\left|\gamma_{i, j}\right|=1$, из (3.5) имеем $\bar{S}_{1, i, j}=S_{0, i, j}$, а эти элементы равны нулю по предыдущему рассуждению. Если же $\left|\gamma_{i, j}\right| \geqslant 2$, то из (3.5) имеем $\bar{S}_{1, i, j}=S_{-\left|\gamma_{i, j}\right|+1, i, j}$, и эти элементы также равны нулю, так как первый индекс $1-\left|\gamma_{i, j}\right|$ отрицателен. Следовательно, $\bar{S}_{1}$ - верхнетреугольная матрица. Аналогично проверяется, что $S_{1}$ - нижнетреугольная матрица.

Далее сравниваем коэффициенты при $\lambda$ в равенстве (3.8) и находим, что

$$
D_{n}\left(S_{0}\right) h_{1}+D_{n}\left(S_{1}\right) h_{0}=D_{m}\left(h_{0}\right) \bar{S}_{1}+D_{m}\left(h_{1}\right) \bar{S}_{0} .
$$

Из последнего равенства легко вытекает, что $S_{1}=\bar{S}_{1}$ - диагональная матрица. Продолжая эту процедуру, можно показать, что все коэффициенты ряда $S$ являются диагональными матрицами. Предложение доказано.

Из предложения 2 получаем, что функции $\ln h$ и $\ln S$ являются производящими функциями законов сохранения для динамической системы (3.1):

$$
\left(D_{m}-1\right) \ln h=\left(D_{n}-1\right) \ln S .
$$

Сравнивая коэффициенты при степенях $\lambda$ в равенстве

$$
\begin{aligned}
& \left(D_{n}-1\right)\left(\ln S_{0}+\frac{S_{1}}{S_{0}} \lambda^{-1}+\left(\frac{S_{2}}{S_{0}}-\frac{1}{2}\left(\frac{S_{1}}{S_{0}}\right)^{2}\right) \lambda^{-2}+\cdots\right)= \\
& =\left(D_{m}-1\right)\left(\ln h_{0}+\frac{h_{1}}{h_{0}} \lambda^{-1}+\left(\frac{h_{2}}{h_{0}}-\frac{1}{2}\left(\frac{h_{1}}{h_{0}}\right)^{2}\right) \lambda^{-2}+\cdots\right),
\end{aligned}
$$

легко можно найти законы сохранения.

Рассмотрим систему полудискретных уравнений

$$
E\left(\left[\mathbf{u}_{t}\right],[\mathbf{u}]\right)=0,
$$

связывающую вектор-функцию $\mathbf{u}$, ее производные по $t$, ее сдвиги и сдвиги ее производных. Допустим, что уравнение (3.10) является условием совместности следующей переопределенной системы линейных уравнений:

$$
y(n+1, t)=P([\mathbf{u}], \lambda) Z y(n, t), \quad y_{t}(n, t)=A\left([\mathbf{u}],\left[\mathbf{u}_{t}\right], \lambda\right) y(n, t) .
$$

Легко проверить, что данное условие совместности влечет равенство

$$
P_{t} Z=D_{n}(A) P Z-P Z A,
$$

равносильное равенству $\left[D_{t}-A, L\right]=0$, где $L=D_{n}^{-1} P Z$, а через $D_{t}$ обозначен оператор полного дифференцирования по $t$. Пусть потенциал $P$ удовлетворяет условиям предложения 1 . Тогда существует формальный ряд $T$ такой, что преобразование сопряжения $x \mapsto T^{-1} x T$ приводит уравнение $\left[D_{t}-A, L\right]=0$ к виду

$$
\left[D_{t}-A_{0}, L_{0}\right]=0,
$$

где $A_{0}=-T^{-1} T_{t}+T^{-1} A T$ - формальный ряд с диагональными коэффициентами, оператор $L_{0}=D_{n}^{-1} h Z=T^{-1} L T$ определен выше в предложении 1. Ясно, что уравнение (3.11) определяет законы сохранения динамической системы (3.10):

$$
D_{t} \ln h=\left(D_{n}-1\right) A_{0} .
$$




\section{4. ПРИМЕРЫ ДИСКРЕТНЫХ МОДЕЛЕЙ}

В примерах настоящего раздела мы покажем, как привести линейную дискретную систему к специальному виду (2.1), используя факторизацию (2.5).

4.1. Дискретное НУШ с производной. В качестве примера, иллюстрирующего применение формальной диагонализации для построения законов сохранения, рассмотрим систему дискретных уравнений

$$
u_{0,1}-u_{1,0}+u_{1,1}\left(\left(u_{0,1}-u_{1,0}\right) v_{1,0}+\varepsilon\right)=0, \quad v_{1,0}-v_{0,1}+v\left(\left(v_{1,0}-v_{0,1}\right) u_{0,1}+\varepsilon\right)=0,
$$

изучавшуюся ранее в работах [12], [19]. Здесь и далее мы используем обозначения $u_{0,1}=D_{m} u, u_{1,0}=D_{n} u, u_{1,1}=D_{n} D_{m} u, u_{-1,1}=D_{n}^{-1} D_{m} u$ и т. д., а также аналогичные обозначения для функций $v, y$ и других.

В разделе 7 мы покажем, что система (4.1) является дискретным аналогом НУШ с производной. Эта система является условием совместности следующей пары линейных уравнений:

$$
y_{1,0}=f y, \quad y_{0,1}=g y .
$$

Другими словами, система (4.1) - это условие коммутирования двух дискретных операторов $L=D_{n}^{-1} f$ и $M=D_{m}^{-1} g$, где

$$
f=\left(\begin{array}{cc}
\lambda+u v & v \\
u & 1
\end{array}\right), \quad g=\left(\begin{array}{cc}
\lambda+\varepsilon+u_{-1,1} v & v \\
u_{-1,1} & 1
\end{array}\right) .
$$

Оператор $L$ легко приводится к специальному виду $L=D_{n}^{-1} P(\lambda) Z$. Для этого достаточно положить $f=P Z$. При этом функция $P(\lambda)$ аналитична на бесконечности по $\lambda$, а главные миноры матрицы $P(\infty)$ отличны от нуля,

$$
P=\left(\begin{array}{cc}
1+u v \lambda^{-1} & v \\
u \lambda^{-1} & 1
\end{array}\right), \quad Z=\left(\begin{array}{ll}
\lambda & 0 \\
0 & 1
\end{array}\right) .
$$

В силу предложения 1 существуют формальные ряды

$$
T=T_{0}+T_{1} \lambda^{-1}+\cdots, \quad h=h_{0}+h_{1} \lambda^{-1}+\cdots
$$

такие, что $L_{0}:=T^{-1} L T$ будет диагональным оператором вида $L_{0}=D^{-1} h Z$. Чтобы найти $T$ и $h$, воспользуемся уравнением $D_{n}(T) h=P \bar{T}$, где

$$
\bar{T}=Z T Z^{-1}=\bar{T}_{0}+\bar{T}_{1} \lambda^{-1}+\cdots
$$

Приравнивая коэффициенты при одинаковых степенях $\lambda$, получим уравнения

$$
\begin{aligned}
D_{n}\left(T_{0}\right) h_{0} & =P_{0} \bar{T}_{0}, \\
D_{n}\left(T_{1}\right) h_{0}+D_{n}\left(T_{0}\right) h_{1}-P_{0} \bar{T}_{1} & =P_{1} \bar{T}_{0}, \\
D_{n}\left(T_{k}\right) h_{0}+D_{n}\left(T_{0}\right) h_{k}-P_{0} \bar{T}_{k} & =P_{1} \bar{T}_{k-1}-\sum_{j=1}^{k-1} D_{n}\left(T_{k-j}\right) h_{j}, \quad k \geqslant 2 .
\end{aligned}
$$


Здесь первое уравнение решается с помощью формулы разложения Гаусса. Из уравнения с номером $k$ можно найти нижнетреугольные части матриц $D_{n}\left(T_{k}\right)$, верхнетреугольные части матриц $\bar{T}_{k}$ и диагональные матрицы $h_{k}$. Остальные элементы искомых матриц, как показано при доказательстве предложения 1, уже найдены на предыдущих шагах (при решении уравнений с меньшими номерами). В результате мы получаем

$$
T=\left(\begin{array}{ll}
1 & 0 \\
0 & 1
\end{array}\right)+\left(\begin{array}{cc}
0 & -v \\
u_{-1,0} & 0
\end{array}\right) \lambda^{-1}+\left(\begin{array}{cc}
0 & u v^{2}-v_{1,0} \\
u_{-2,0}-u_{-1,0}^{2} v_{-1,0} & 0
\end{array}\right) \lambda^{-2}+\cdots
$$

и

$$
h=\left(\begin{array}{ll}
1 & 0 \\
0 & 1
\end{array}\right)+\left(\begin{array}{cc}
u v & 0 \\
0 & -u v
\end{array}\right) \lambda^{-1}+\left(\begin{array}{cc}
u_{-1,0} v & 0 \\
0 & u^{2} v^{2}-u v_{1,0}
\end{array}\right) \lambda^{-2}+\cdots .
$$

Второй оператор диагонализуется следующим образом

$$
\begin{aligned}
& S:=D_{m}\left(T^{-1}\right) g T=\left(\begin{array}{cc}
1 & 0 \\
0 & 0
\end{array}\right) \lambda+\left(\begin{array}{cc}
\varepsilon+u_{-1,1} v & 0 \\
0 & 1
\end{array}\right)+\left(\begin{array}{cc}
u_{-1,0} v & 0 \\
0 & -u_{-1,1} v
\end{array}\right) \lambda^{-1}+ \\
& +\left(\begin{array}{cc}
u_{-2,0} v-u_{-1,0}^{2} v v_{-1,0} & 0 \\
0 & u_{-1,1}^{2} v^{2}+u_{-1,1} v \varepsilon-u_{-1,1} v_{0,1}
\end{array}\right) \lambda^{-2}+\cdots .
\end{aligned}
$$

Выпишем три закона сохранения из бесконечной последовательности, получающейся в результате диагонализации (см. также работу [12]):

$$
\begin{aligned}
\left(D_{n}-1\right) u_{-1,1} v & =\left(D_{m}-1\right) u v, \\
\left(D_{n}-1\right)\left(u_{-1,0} v-\frac{1}{2}\left(\varepsilon+u_{-1,1} v\right)^{2}\right) & =\left(D_{m}-1\right)\left(u_{-1,0} v-\frac{1}{2} u^{2} v^{2}\right), \\
\left(D_{n}-1\right)\left(\frac{1}{2} u_{0,1}^{2} v_{1,0}^{2}+u_{0,1} v_{1,0} \varepsilon-u_{0,1} v_{1,1}\right) & =\left(D_{m}-1\right)\left(\frac{1}{2} u_{1,0}^{2} v_{1,0}^{2}-u_{1,0} v_{2,0}\right) .
\end{aligned}
$$

\section{2. Обобщенная дискретная цепочка Тоды, соответствующая алгебре} Ли $A_{1}^{(1)}$. Рассмотрим найденную в работе [20] систему уравнений, которая соответствует аффинной алгебре Ли $A_{1}^{(1)}$ :

$$
u u_{1,1}-u_{1,0} u_{0,1}=v_{0,1}^{2}, \quad v v_{1,1}-v_{1,0} v_{0,1}=u_{1,0}^{2} .
$$

Данная система является интегрируемой дискретизацией цепочки Тоды, связанной с алгеброй $A_{1}^{(1)}$,

$$
r_{x, y}=e^{-2 r+2 s}, \quad s_{x, y}=e^{2 r-2 s} .
$$

Известно, что система (4.5) допускает пару Лакса

$$
\Psi_{1,0}=f \Psi, \quad \Psi_{0,1}=g \Psi,
$$

где

$$
f=\left(\begin{array}{cc}
\frac{u v_{1,0}}{u_{1,0} v}+\lambda & -\frac{u_{1,0} v_{-1,0}}{u v} \\
-\lambda & \frac{u_{1,0} v_{-1,0}}{u v}
\end{array}\right), \quad g=\left(\begin{array}{cc}
1+\frac{1}{\lambda} \frac{u v_{0,1}}{u_{0,1} v} & \frac{1}{\lambda} \frac{v_{0,1}^{2} v_{-1,0}}{u u_{0,1} v} \\
\frac{u^{2}}{v v_{-1,1}} & \frac{v_{0,1} v_{-1,0}}{v v_{-1,1}}
\end{array}\right)
$$


Для удобства положим

$$
\begin{gathered}
a=\frac{v_{1,0} u}{v u_{1,0}}, \quad b=\frac{u_{1,0} v_{-1,0}}{u v}, \quad c=\frac{v_{0,1} u}{u_{0,1} v}, \\
d=\frac{v_{0,1}^{2} v_{-1,0}}{u u_{0,1} v}, \quad e=\frac{u^{2}}{v v_{-1,1}}=\frac{v_{0,1} v_{-1,0}}{v v_{-1,1}}-1 .
\end{gathered}
$$

Тогда непосредственно можно проверить, что $c(1+e)=e d$, а отсюда $\operatorname{det} g=1+e$. Итак, мы имеем

$$
f=\left(\begin{array}{cc}
a+\lambda & -b \\
-\lambda & b
\end{array}\right), \quad g=\left(\begin{array}{cc}
1+c \lambda^{-1} & d \lambda^{-1} \\
e & 1+e
\end{array}\right) .
$$

С целью привести первое из уравнений (4.6) к виду (2.1) разложим матрицу $f$ в произведение вида $f=\alpha Z \gamma$, где

$$
\alpha=\left(\begin{array}{cc}
1+a \lambda^{-1} & 0 \\
-1 & \frac{a}{1+a \lambda^{-1}}
\end{array}\right), \quad Z=\left(\begin{array}{cc}
\lambda & 0 \\
0 & \lambda^{-1}
\end{array}\right), \quad \gamma=\left(\begin{array}{cc}
1 & -\frac{b \lambda^{-1}}{1+a \lambda^{-1}} \\
0 & b
\end{array}\right) .
$$

Совершая замену $\phi=\gamma \Psi$, получим требуемую специальную форму $\phi_{1,0}=P(\lambda) Z \phi$ первого уравнения в (4.6), где

$$
P(\lambda)=\gamma_{1,0} \alpha=\left(\begin{array}{cc}
1+a \lambda^{-1}+\frac{b_{1,0} \lambda^{-1}}{1+a_{1,0} \lambda^{-1}} & -\frac{a b_{1,0} \lambda^{-1}}{\left(1+a_{1,0} \lambda^{-1}\right)\left(1+a \lambda^{-1}\right)} \\
-b_{1,0} & \frac{a b_{1,0}}{1+a \lambda^{-1}}
\end{array}\right) .
$$

Диагонализуем оператор $M=D_{m}^{-1} g$ и приведем его к виду $M_{0}=T^{-1} \gamma M \gamma^{-1} T$, при этом $M_{0}=D_{m}^{-1} S$, где $S=D_{m}\left(T^{-1} \gamma\right) g \gamma^{-1} T$ или, эквивалентно,

$$
\begin{aligned}
S= & \left(\begin{array}{cc}
1 & 0 \\
0 & \frac{u u_{1,1}}{u_{1,0} u_{0,1}}
\end{array}\right)+ \\
& +\left(\begin{array}{cc}
-\frac{u u_{1,0}}{v v_{0,1}} & 0 \\
0 & \frac{u^{2} u_{1,1}}{u_{0,1} v v_{0,1}}
\end{array}\right) \lambda^{-1}+\left(\begin{array}{cc}
-\frac{u^{2} v_{1,1}}{v v_{0,1}^{2}} & 0 \\
0 & \frac{u^{3} u_{1,1} v_{1,0}}{u_{1,0} u_{0,1} v^{2} v_{0,1}}
\end{array}\right) \lambda^{-2}+\cdots
\end{aligned}
$$

Уравнение $D_{n}\left(S_{0}\right) / S_{0}=D_{m}\left(h_{0}\right) / h_{0}$ для функций $S, h$ позволяет построить бесконечную серию законов сохранения. Приведем первые четыре из них:

$$
\begin{aligned}
\left(D_{n}-1\right) \ln \frac{u u_{1,1}}{u_{1,0} u_{0,1}} & =\left(D_{m}-1\right) \ln \frac{u u_{2,0}}{u_{1,0}^{2}}, \\
\left(D_{n}-1\right)\left(-\frac{u u_{1,0}}{v v_{0,1}}\right) & =\left(D_{m}-1\right)\left(\frac{u v_{1,0}}{v u_{1,0}}+\frac{v u_{2,0}}{u_{1,0} v_{1,0}}\right), \\
\left(D_{n}-1\right)\left(\frac{u^{2} v_{1,1}}{v v_{0,1}^{2}}-\frac{1}{2}\left(\frac{u u_{1,0}}{v v_{0,1}}\right)^{2}\right) & =\left(D_{m}-1\right)\left(\frac{v v_{2,0}}{v_{1,0}^{2}}-\frac{1}{2}\left(\frac{u v_{1,0}}{u_{1,0} v}+\frac{u_{2,0} v}{u_{1,0} v_{1,0}}\right)^{2}\right), \\
\left(D_{n}-1\right)\left(-\frac{u^{2} v_{1,0}}{v^{2} v_{0,1}}-\frac{1}{2}\left(\frac{u u_{1,0}}{v v_{0,1}}\right)^{2}\right) & =\left(D_{m}-1\right)\left(\frac{v u_{2,0}}{u_{1,0} v_{1,0}}+\frac{1}{2}\left(\frac{u v_{1,0}}{u_{1,0} v}+\frac{u_{2,0} v}{u_{1,0} v_{1,0}}\right)^{2}\right) .
\end{aligned}
$$


Потенциалы $f, g$ в (4.6) имеют две точки сингулярности $\lambda=0, \lambda=\infty$, поэтому система (4.5) допускает две последовательности законов сохранения. Для построения второй серии законов сохранения воспользуемся специальной формой оператора $M$, которую легко можно найти, используя разложение

$$
g(\lambda)=\left(\begin{array}{cc}
c+\xi^{-1} & 0 \\
e \xi^{-1} & 1+e
\end{array}\right) Z(\xi)\left(\begin{array}{cc}
1 & \frac{d}{c+\xi^{-1}} \\
0 & \frac{1}{c+\xi^{-1}}
\end{array}\right)
$$

где $\xi=\lambda^{-1}, Z(\xi)=\operatorname{diag}\left(\xi, \xi^{-1}\right)$. В результате имеем

$$
\begin{aligned}
\left(D_{n}-1\right) \ln \frac{u v_{1,0}}{u_{1,0} v} & =\left(D_{m}-1\right) \ln \frac{u v_{0,1}}{u_{0,1} v}, \\
\left(D_{n}-1\right)\left(\frac{u v_{0,2}}{u_{0,1} v_{0,1}}+\frac{u_{0,1} v}{u v_{1,0}}\right) & =\left(D_{m}-1\right)\left(\frac{u_{1,0} v}{u v_{1,0}}-\frac{v^{2} v_{1,1}}{u u_{1,0} v_{1,0}}\right), \\
\left(D_{n}-1\right) \ln \frac{u_{0,2} v_{-1,0} v_{0,1}^{2}}{u_{0,1} v v_{-1,1} v_{0,2}} & =\left(D_{m}-1\right) \ln \frac{u_{1,1} v_{1,0} v_{0,1} v_{-1,0}}{u_{0,1} v^{2} v_{1,1}} .
\end{aligned}
$$

4.3. Дискретное потенциированное уравнение Кортевега-де Фриза. Рассмотрим дискретное потенциированное уравнение Кортевега-де Фриза [21]

$$
\left(u_{1,1}-u\right)\left(u_{1,0}-u_{0,1}\right)=4 c^{2} .
$$

Бесконечная серия законов сохранения для него была получена в работе [22], иерархия высших симметрий описана в статье [23]. Пара Лакса для уравнения (4.7) задается в виде системы линейных дискретных уравнений [24]

$$
\Psi_{1,0}=f \Psi, \quad \Psi_{0,1}=g \Psi
$$

где потенциалы $f, g$ полиномиальны относительно спектрального параметра и записываются следующим образом:

$$
f=\left(\begin{array}{cc}
-u_{1,0} & 1 \\
-\lambda^{-2}-u u_{1,0} & u
\end{array}\right), \quad g=\left(\begin{array}{cc}
-u_{0,1} & 1 \\
-\lambda^{-2}+4 c^{2}-u u_{0,1} & u
\end{array}\right) .
$$

Положим $\Psi=\lambda^{-n} \psi$ и получим $\psi_{1,0}=F \psi$, где потенциал

$$
F=\left(\begin{array}{cc}
-u_{1,0} \lambda & \lambda \\
-\lambda^{-1}-u u_{1,0} \lambda & u \lambda
\end{array}\right)
$$

имеет единичный определитель. Приведем уравнение $\psi_{1,0}=F \psi$ к виду (2.1). Для этого разложим потенциал $F$ в произведение $F=\alpha Z \gamma$ треугольных и диагональной матриц, где

$$
\alpha=\left(\begin{array}{cc}
1 & 0 \\
u+\frac{\lambda^{-2}}{u_{1,0}} & -\frac{1}{u_{1,0}}
\end{array}\right), \quad Z=\left(\begin{array}{cc}
\lambda & 0 \\
0 & \lambda^{-1}
\end{array}\right), \quad \gamma=\left(\begin{array}{cc}
-u_{1,0} & 1 \\
0 & 1
\end{array}\right) .
$$


Делая замену $y=\gamma \psi$, перепишем уравнение в требуемой форме $y_{1,0}=P(\lambda) Z y$, где $P(\lambda)=\gamma_{1,0} \alpha$ или, в координатном представлении,

$$
P(\lambda)=\left(\begin{array}{cc}
u-u_{2,0}+\frac{\lambda^{-2}}{u_{1,0}} & -\frac{1}{u_{1,0}} \\
u+\frac{1}{u_{1,0}} \lambda^{-2} & -\frac{1}{u_{1,0}}
\end{array}\right)=\left(\begin{array}{cc}
u-u_{2,0} & -\frac{1}{u_{1,0}} \\
u & -\frac{1}{u_{1,0}}
\end{array}\right)+\left(\begin{array}{cc}
\frac{1}{u_{1,0}} & 0 \\
\frac{1}{u_{1,0}} & 0
\end{array}\right) \lambda^{-2} .
$$

В силу предложения 1 оператор $L=D_{n}^{-1} P(\lambda) Z$ можно привести к диагональному виду. Найдем соответствующие формальные ряды $T$ и $h$ :

$$
\begin{aligned}
T= & \left(\begin{array}{cc}
1 & 0 \\
\frac{u_{-1,0}}{u_{-1,0}-u_{1,0}} & 1
\end{array}\right)+\left(\begin{array}{cc}
0 & \frac{1}{u_{1,0}\left(u-u_{2,0}\right)} \\
\frac{u_{1,0}}{\left(u_{-1,0}-u_{1,0}\right)^{2}\left(u_{-2,0}-u\right)} & 0
\end{array}\right) \lambda^{-2}+\cdots \\
h= & \left(\begin{array}{cc}
u-u_{2,0} & 0 \\
0 & \frac{u_{2,0}}{u_{1,0}\left(u-u_{2,0}\right)}
\end{array}\right)+ \\
& +\left(\begin{array}{cc}
-\frac{1}{u_{-1,0}-u_{1,0}} & \frac{u u_{1,0}-u_{1,0} u_{2,0}+u_{2,0} u_{3,0}}{u_{1,0}^{2}\left(u-u_{2,0}\right)^{2}\left(u_{1,0}-u_{3,0}\right)}
\end{array}\right) \lambda^{-2}+\cdots
\end{aligned}
$$

Здесь $L=T L_{0} T^{-1}, L_{0}=D_{n}^{-1} h Z$. Второму уравнению пары после указанной выше замены переменных будет соответствовать оператор $M=D_{m}^{-1} \gamma_{0,1} g \gamma$. Положим $M_{0}=T^{-1} M T$, тогда $M_{0}=D_{m}^{-1} S$, где $S$ - формальный ряд, вычисленный по формуле $S=D_{m}\left(T^{-1}\right) \gamma_{0,1} g \gamma^{-1} T$. Выпишем первые слагаемые ряда $S$ в явном виде:

$$
\begin{aligned}
S= & \left(\begin{array}{cc}
u-u_{1,1} & 0 \\
0 & \frac{u_{1,1}\left(u_{1,0}-u_{0,1}\right)}{u_{1,0}}
\end{array}\right)+ \\
& +\left(\begin{array}{cc}
\frac{\left(u-u_{-1,1}\right)\left(u_{-1,0}-u_{1,0}\right)+\left(u_{2,1}-u_{1,0}\right)\left(u_{-1,1}-u_{1,1}\right)}{\left(u_{-10}-u_{10}\right)\left(u_{-11}-u_{1,1}\right)\left(u_{0,1}-u_{2,1}\right)} & 0 \\
0 & s_{22}
\end{array}\right) \lambda^{-2}+\cdots
\end{aligned}
$$

Здесь выражение $s_{22}$ опущено, посколько оно является слишком громоздким. Отметим, что формальные ряды $T, h, S$ были найдены ранее другим способом в работе [13].

В результате несложных вычислений находим законы сохранения. Первые три из них представлены ниже (см. также работу [22]):

$$
\begin{gathered}
\left(D_{n}-1\right) \ln \left(u-u_{1,1}\right)=\left(D_{m}-1\right) \ln \left(u-u_{2,0}\right), \\
\left(D_{n}-1\right) \ln \frac{u_{1,1}\left(u_{1,0}-u_{0,1}\right)}{u_{1,0}}=\left(D_{m}-1\right) \ln \frac{u_{2,0}}{u_{1,0}\left(u-u_{2,0}\right)}, \\
\left(D_{n}-1\right) \frac{\left(u-u_{-1,1}\right)\left(u_{-1,0}-u_{1,0}\right)+\left(u_{2,1}-u_{1,0}\right)\left(u_{-1,1}-u_{1,1}\right)}{\left(u_{-1,0}-u_{1,0}\right)\left(u_{-1,1}-u_{1,1}\right)\left(u_{1,1}-u\right)\left(u_{2,1}-u_{0,1}\right)}= \\
=\left(D_{m}-1\right) \frac{1}{\left(u_{-1,0}-u_{1,0}\right)\left(u_{2,0}-u\right)} .
\end{gathered}
$$


Ясно, что пара Лакса (4.8) может быть диагонализована также в окрестности особой точки $\xi=0$ второго из операторов пары, где $\xi=\lambda^{-2}+4 c^{2}$.

4.4. Одевающая цепочка Леви-Ямилова. Рассмотрим дискретное уравнение из работы [25]:

$$
\left(u_{1,0}+\alpha\right)(u-\alpha)=\left(u_{1,1}-\alpha_{1}\right)\left(u_{0,1}+\alpha_{1}\right),
$$

где $u=u(n, m)$ - искомая функция, $\alpha=\alpha(m)$ - заданная функция и $\alpha_{1}=\alpha(m+1)$, аналогичное обозначение мы будем использовать и для других сдвинутых функций одной переменной. Уравнение (4.9) имеет $L-A$-пару

$$
\Psi_{1,0}=f \Psi, \quad \Psi_{0,1}=g \Psi,
$$

где для $v=\left(u_{1,1}+\alpha_{1}\right)\left(u_{0,1}-\alpha_{1}\right)$ имеем

$$
f=\left(\begin{array}{cc}
\lambda & -v \\
1 & 0
\end{array}\right), \quad g=\frac{1}{u_{0,1}-\alpha_{1}}\left(\begin{array}{cc}
\lambda\left(u_{0,1}-\alpha_{1}\right) & 2 \alpha_{1}\left(u_{0,1}^{2}-\alpha_{1}^{2}\right) \\
-2 \alpha_{1} & \lambda\left(u_{0,1}+\alpha_{1}\right)
\end{array}\right) .
$$

Для приведения первого из уравнений (4.10) к виду (2.1) запишем потенциал $f$ в виде произведения $f=\beta Z \gamma$, где

$$
\beta=\left(\begin{array}{cc}
1 & 0 \\
\lambda^{-1} & v
\end{array}\right), \quad Z=\left(\begin{array}{cc}
\lambda & 0 \\
0 & \lambda^{-1}
\end{array}\right), \quad \gamma=\left(\begin{array}{cc}
1 & v \lambda^{-1} \\
0 & 1
\end{array}\right) .
$$

Следуя общей схеме, получаем

$$
P=\gamma_{1,0} \beta=\left(\begin{array}{cc}
1-v_{1,0} \lambda^{-2} & -v v_{1,0} \lambda^{-2} \\
\lambda^{-1} & v
\end{array}\right) .
$$

Матрица $P$ удовлетворяет условиям предложения 1 , поэтому из уравнения $(2.8)$ можно найти формальные ряды $T$ и $h$ :

$$
\begin{aligned}
T= & \left(\begin{array}{ll}
1 & 0 \\
0 & 1
\end{array}\right)+\left(\begin{array}{ll}
0 & 0 \\
1 & 0
\end{array}\right) \lambda^{-1}+\left(\begin{array}{cc}
0 & v v_{1,0} \\
v+v_{-1,0} & 0
\end{array}\right) \lambda^{-3}+ \\
& +\left(\begin{array}{cc}
0 & v v_{1,0} v_{2,0}+v v_{1,0}^{2} \\
v v_{-1,0}+\left(v+v_{1,0}\right)^{2} & 0
\end{array}\right) \lambda^{-5}+\cdots \\
h= & \left(\begin{array}{ll}
1 & 0 \\
0 & v
\end{array}\right)+\left(\begin{array}{cc}
-v_{1,0} & 0 \\
0 & v v_{1,0}
\end{array}\right) \lambda^{-2}+\left(\begin{array}{cc}
-v v_{1,0} & 0 \\
0 & v v_{1,0} v_{2,0}+v v_{1,0}^{2}
\end{array}\right) \lambda^{-4}+\cdots .
\end{aligned}
$$

Диагонализуя второй оператор $L-A$-пары, найдем

$$
\begin{aligned}
S= & D_{m}\left(T^{-1} \gamma\right) g \gamma^{-1} T=\left(\begin{array}{cc}
1 & 0 \\
0 & \frac{u_{0,1}+\alpha_{1}}{u_{0,1}-\alpha_{1}}
\end{array}\right) \lambda+ \\
& +\left(\begin{array}{cc}
2 \alpha_{1}\left(u_{0,1}+\alpha_{1}\right) & 0 \\
0 & -\frac{2 \alpha_{1} v}{u_{0,1}-\alpha_{1}}
\end{array}\right) \lambda^{-1}+\left(\begin{array}{cc}
-\frac{2 \alpha_{1} v v_{0,1}}{u_{0,1}-\alpha_{1}} & 0 \\
0 & \frac{2 \alpha_{1} v v_{1,0}}{u_{0,1}-\alpha_{1}}
\end{array}\right) \lambda^{-3}+\cdots
\end{aligned}
$$


Далее, приравнивая коэффициенты при степенях $\lambda$ в выражении вида (3.9), получаем бесконечную последовательность законов сохранения. Первые из них выписаны ниже:

$$
\begin{aligned}
\left(D_{n}-1\right) \ln \frac{u_{0,1}+\alpha_{1}}{u_{0,1}-\alpha_{1}} & =\left(D_{m}-1\right) \ln v \\
\left(D_{n}-1\right)\left(2 \alpha_{1}\left(u_{0,1}+\alpha_{1}\right)\right) & =\left(D_{m}-1\right)(-v), \\
\left(D_{n}-1\right) \frac{-2 \alpha_{1} v}{u_{0,1}+\alpha_{1}} & =\left(D_{m}-1\right) v_{1,0}, \\
\left(D_{n}-1\right)\left(\frac{2 \alpha_{1} v v_{1,0}}{u_{0,1}+\alpha_{1}}-\frac{2 \alpha_{1}^{2} v^{2}}{\left(u_{0,1}+\alpha_{1}\right)^{2}}\right) & =\left(D_{m}-1\right)\left(v_{1,0} v_{2,0}+\frac{1}{2} v_{1,0}^{2}\right) .
\end{aligned}
$$

\section{5. ПРИМЕРЫ ПОЛУДИСКРЕТНЫХ МОДЕЛЕЙ}

5.1. Цепочка Тоды. Рассмотрим хорошо известную цепочку Тоды

$$
\ddot{q}=e^{q_{1}-q}-e^{q-q-1}
$$

как пример применения диагонализации к дифференциально-разностным уравнениям. Уравнение (5.1) является условием совместности следующих линейных уравнений:

$$
\Psi_{1}=f \Psi, \quad \Psi_{t}=g \Psi
$$

Здесь потенциалы полиномиальны относительно спектрального параметра $\lambda$ и имеют вид

$$
f=\left(\begin{array}{cc}
p+\lambda & e^{q} \\
-e^{-q} & 0
\end{array}\right), \quad g=\left(\begin{array}{cc}
0 & -e^{q} \\
e^{-q-1} & \lambda
\end{array}\right)
$$

где $p=\dot{q}$. Как и в предыдущих примерах, для получения особой формы первого оператора Лакса разложим потенциал $f$ в произведение $f=\alpha Z \gamma$, где

$$
\alpha=\left(\begin{array}{cc}
1 & 0 \\
-\frac{e^{-q}}{\lambda+p} & \frac{1}{1+p \lambda^{-1}}
\end{array}\right), \quad Z=\left(\begin{array}{cc}
\lambda & 0 \\
0 & \lambda^{-1}
\end{array}\right), \quad \gamma=\left(\begin{array}{cc}
1+p \lambda^{-1} & e^{q} \lambda^{-1} \\
0 & 1
\end{array}\right)
$$

Используя замену переменной $\phi=\gamma \Psi$, преобразуем первое уравнение в $(5.2)$ к виду $\phi_{1,0}=P(\lambda) Z \phi$, где

$$
\begin{aligned}
P(\lambda) & =\gamma_{1} \alpha=\left(\begin{array}{cc}
1+p_{1} \lambda^{-1}-\frac{e^{q_{1}-q} \lambda^{-2}}{1+p \lambda^{-1}} & \frac{e^{q_{1} \lambda^{-1}}}{1+p \lambda^{-1}} \\
-\frac{e^{-q} \lambda^{-1}}{1+p \lambda^{-1}} & \frac{1}{1+p \lambda^{-1}}
\end{array}\right)= \\
& =1+\left(\begin{array}{cc}
p_{1} & e^{q_{1}} \\
-e^{-q} & -p
\end{array}\right) \lambda^{-1}+\left(\begin{array}{cc}
-e^{q_{1}-q} & -e^{q_{1}} p \\
e^{-q} p & p^{2}
\end{array}\right) \lambda^{-2}+\left(\begin{array}{cc}
e^{q_{1}-q} p & e^{q_{1}} p^{2} \\
-e^{-q} p^{2} & -p^{3}
\end{array}\right) \lambda^{-3}+\cdots .
\end{aligned}
$$


Очевидно, функция $P(\lambda)$ является аналитической в окрестности точки $\lambda=\infty$ и $\operatorname{det}_{j} P(\infty) \neq 0$. Решая уравнение $D_{n} Y h=P Z Y Z^{-1}$, находим формальные ряды $Y$ и $h$ :

$$
\begin{aligned}
Y= & \left(\begin{array}{ll}
1 & 0 \\
0 & 1
\end{array}\right)+\left(\begin{array}{cc}
0 & 0 \\
-e^{-q_{-1}} & 0
\end{array}\right) \lambda^{-1}+\left(\begin{array}{cc}
0 & 0 \\
e^{-q_{-1}}\left(p+p_{-1}\right) & 0
\end{array}\right) \lambda^{-2}+ \\
& +\left(\begin{array}{cc}
0 \\
-e^{-q_{-1}}\left(p^{2}+p p_{-1}+p_{-1}^{2}\right)-e^{-q_{-2}}-e^{q-2 q_{-1}} & 0
\end{array}\right) \lambda^{-3}+\cdots, \\
h= & \left(\begin{array}{ll}
1 & 0 \\
0 & 1
\end{array}\right)+\left(\begin{array}{cc}
p_{1} & 0 \\
0 & -p
\end{array}\right) \lambda^{-1}+\left(\begin{array}{cc}
-e^{q_{1}-q} & 0 \\
0 & p^{2}+e^{q_{1}-q}
\end{array}\right) \lambda^{-2}+\cdots .
\end{aligned}
$$

Диагонализуем второй оператор в (5.2), используя формальный ряд $T=\gamma^{-1} Y$, где

$$
\begin{aligned}
& T=\left(\begin{array}{ll}
1 & 0 \\
0 & 1
\end{array}\right)+\left(\begin{array}{cc}
-p & -e^{q} \\
-e^{-q_{-1}} & 0
\end{array}\right) \lambda^{-1}+\left(\begin{array}{cc}
p^{2}+e^{q-q_{-1}} & e^{q} p \\
e^{-q_{-1}}\left(p+p_{-1}\right) & 0
\end{array}\right) \lambda^{-2}+ \\
& +\left(\begin{array}{cc}
-p^{3}-e^{q-q_{-1}}\left(2 p+p_{-1}\right) & -e^{q} p^{2}-e^{q_{1}} \\
-e^{-q_{-2}}-e^{-q_{-1}}\left(p^{2}+p p_{-1}+p_{-1}^{2}\right)-e^{q-2 q_{-1}} & 0
\end{array}\right) \lambda^{-3}+\cdots .
\end{aligned}
$$

Тогда из уравнений (5.2) получаем

$$
\begin{aligned}
S:= & -T^{-1} T_{t}+T^{-1} g T=\left(\begin{array}{ll}
0 & 0 \\
0 & 1
\end{array}\right) \lambda-\left(\begin{array}{cc}
1 & 0 \\
0 & 1
\end{array}\right)+\left(\begin{array}{cc}
e^{q_{1}-q} & 0 \\
0 & -e^{q-q_{-1}}
\end{array}\right) \lambda^{-1}+ \\
& +\left(\begin{array}{cc}
-e^{q_{1}-q} p & 0 \\
0 & e^{q-q_{-1}} p
\end{array}\right) \lambda^{-2}+\left(\begin{array}{cc}
e^{2 q-2 q_{-1}}+e^{q_{1}-q} p^{2} & 0 \\
0 & s_{2,2}
\end{array}\right) \lambda^{-3}+\cdots,
\end{aligned}
$$

где

$$
s_{2,2}=e^{q-q_{-2}}+e^{2 q-2 q_{-1}}+2 e^{q_{1}-q_{-1}}+e^{q-q-1}\left(p_{-1}^{2}+2 p^{2}+p p_{-1}\right) .
$$

В итоге получаем законы сохранения для цепочки Тоды (см. также работу [26]):

$$
\begin{aligned}
D_{t} p_{1} & =\left(D_{n}-1\right) e^{q_{1}-q}, \\
D_{t}\left(\frac{p_{1}^{2}}{2}+e^{q_{1}-q}\right) & =\left(D_{n}-1\right) e^{q_{1}+q} p, \\
D_{t}\left(\frac{p^{2}}{2}-e^{q_{1}-q}\right) & =\left(D_{n}-1\right)\left(-e^{q-q_{-1}} p\right), \\
D_{t}\left(-\frac{p^{3}}{3}-e^{q_{1}-q}\left(p_{1}+p\right)\right) & =\left(D_{n}-1\right)\left(e^{2 q-2 q_{-1}}+e^{q_{1}-q} p^{2}\right) .
\end{aligned}
$$

5.2. Одевающая цепочка Веселова-Шабата-Ямилова. Последовательное применение преобразования Беклунда к уравнению Кортевега-де Фриза порождает полудискретное уравнение [27]-[29]

$$
f_{1, x}+f_{x}=f^{2}-f_{1}^{2}+\alpha-\alpha_{1} \quad \text { или } \quad u_{1, x}+u_{x}=\left(u_{1}-u\right) \sqrt{2\left(u_{1}+u\right)-4 \alpha},
$$

где

$$
u=f^{2}+f_{x}+\alpha, \quad u_{1}=f^{2}-f_{x}+\alpha .
$$


Уравнение (5.3) допускает пару Лакса

$$
\Psi_{x}=U \Psi, \quad \Psi_{1}=W \Psi
$$

где

$$
U=\left(\begin{array}{cc}
0 & 1 \\
u+\lambda^{2} & 0
\end{array}\right), \quad W=\left(\begin{array}{cc}
-f & 1 \\
f^{2}+\alpha+\lambda^{2} & -f
\end{array}\right)
$$

Диагонализацию пары Лакса удобнее начать с первого из уравнений (5.4). Отметим, что главный член потенциала $U$, т. е. коэффициент перед $\lambda^{2}$, имеет равные собственные значения $\lambda_{1}=\lambda_{2}=0$. В этом случае метод диагонализации невозможно применить непосредственно к системе (5.4). Поэтому сначала совершим замену переменных $\Psi=K \phi$, где

$$
K=\left(\begin{array}{cc}
1 & 1 \\
\lambda & -\lambda
\end{array}\right)
$$

и приведем $L-A$-пару к виду

$$
\phi_{x}=\bar{U} \phi, \quad \phi_{1}=\bar{W} \phi,
$$

где

$$
\begin{gathered}
\bar{U}=\sigma_{3} \lambda+\frac{u}{2} R \lambda^{-1}, \quad \bar{W}=\sigma_{3} \lambda-f+\frac{f^{2}+\alpha}{2} R \lambda^{-1}, \\
\sigma_{3}=\left(\begin{array}{cc}
1 & 0 \\
0 & -1
\end{array}\right), \quad R=\left(\begin{array}{cc}
1 & 1 \\
-1 & -1
\end{array}\right) .
\end{gathered}
$$

Теперь коэффициент при $\lambda$ в операторе $\bar{U}$ имеет разные собственные значения \pm 1 , и мы можем применить метод формальной диагонализации к первому уравнению в (5.5). Отметим, что формальный ряд $T$ диагонализует оператор $D_{x}-\bar{U}$ так, что $D_{x}-h=T^{-1}\left(D_{x}-\bar{U}\right) T$. Неизвестные ряды

$$
T=1+T_{1} \lambda^{-1}+T_{2} \lambda^{-2}+\cdots, \quad h=\sigma_{3} \lambda+h_{0}+h_{1} \lambda^{-1}+h_{2} \lambda^{-2}+\cdots
$$

находятся из уравнения

$$
\begin{aligned}
\left(D_{x}-\sigma_{3} \lambda\right. & \left.-\frac{u}{2} R \lambda^{-1}\right)\left(1+T_{1} \lambda^{-1}+T_{2} \lambda^{-2}+\cdots\right)= \\
& =\left(1+T_{1} \lambda^{-1}+T_{2} \lambda^{-2}+\cdots\right)\left(D-\sigma_{3} \lambda-h_{0}-h_{1} \lambda^{-1}-h_{2} \lambda^{-2}-\ldots\right) .
\end{aligned}
$$

Пропустим вычисления и запишем только ответы:

$$
\begin{aligned}
T= & +\lambda^{-1}+\left(\begin{array}{cc}
0 & -\frac{u}{4} \\
-\frac{u}{4} & 0
\end{array}\right) \lambda^{-2}+\left(\begin{array}{cc}
0 & -\frac{u}{4}-\frac{u_{x}}{8} \\
-\frac{u}{4}+\frac{u_{x}}{8} & 0
\end{array}\right) \lambda^{-3}+ \\
& +\left(\begin{array}{cc}
0 & \frac{u^{2}}{8}-\frac{u_{x}}{8}-\frac{u_{x x}}{16} \\
\frac{u^{2}}{8}+\frac{u_{x}}{8}-\frac{u_{x x}}{16} & 0
\end{array}\right) \lambda^{-4}+\cdots,
\end{aligned}
$$




$$
h=\sigma_{3} \lambda+\left(\begin{array}{cc}
\frac{u}{2} & 0 \\
0 & -\frac{u}{2}
\end{array}\right) \lambda^{-1}+\left(\begin{array}{cc}
-\frac{u^{2}}{8} & 0 \\
0 & \frac{u^{2}}{8}
\end{array}\right) \lambda^{-3}+\left(\begin{array}{cc}
\frac{u u_{x}}{16} & 0 \\
0 & \frac{u u_{x}}{16}
\end{array}\right) \lambda^{-4}+\cdots
$$

Второе уравнение в (5.5) приводится к диагональной форме $\psi_{1}=S \psi$ преобразованием $\phi=T \psi$. Формальный ряд $S=T^{-1} \bar{W} T$ принимает вид

$$
S=\sigma_{3} \lambda-f E+\frac{f^{2}+\alpha}{2} \sigma_{3} \lambda^{-1}-\frac{\left(f^{2}+\alpha\right) u}{8} \sigma_{3} \lambda^{-3}+\frac{\left(f^{2}+\alpha\right)\left(f_{x x}+2 f f_{x}\right)}{16} E \lambda^{-4}+\cdots,
$$

где $E$ - единичная матрица. Чтобы найти законы сохранения, используем соотношение $D_{x} \ln S=\left(D_{n}-1\right) h$. Выпишем некоторые из них в явной форме:

$$
\begin{aligned}
D_{x} f & =-\left(D_{n}-1\right) \frac{u}{2}, \\
D_{x}\left(\frac{f^{3}}{3}+\alpha f\right) & =-\left(D_{n}-1\right) \frac{u^{2}}{4}, \\
D_{x} f_{x}\left(f^{2}+\alpha\right) & =-\left(D_{n}-1\right) \frac{u u_{x}}{2} .
\end{aligned}
$$

Отметим, что коэффициенты ряда $S$ зависят от переменных $f, f_{x}, f_{x x}$ и т. д. Рассматриваемая пара Лакса также допускает формальную диагонализацию в окрестности точки $\lambda=0$ с коэффициентами, зависящими только от сдвигов потенциала $f$.

\section{6. ДИАГОНАЛИЗАЦИЯ ОПЕРАТОРА ЛАКСА И ОПИСАНИЕ ВЫСШИХ СИММЕТРИЙ ДИНАМИЧЕСКОЙ СИСТЕМЫ}

В этом разделе мы обсудим связь между диагонализацией дискретного оператора Лакса и высшими симметриями динамической системы. Сосредоточимся на простом случае оператора малой матричной размерности (общий случай вызывает затруднения): пусть

$$
L=D_{n}^{-1} c Z, \quad Z=\operatorname{diag}(\lambda, 1),
$$

где $c=c(n, m)$ - произвольная функция со значениями в группе Ли $G L(2, \mathbb{C})$ невырожденных матриц размера $2 \times 2$. Предположим, что элементы матрицы $c=c(n, m)$ удовлетворяют следующему условию регулярности: $c_{11} \neq 0$. Тогда из предложения 1 следует, что оператор $L$ приводится к формально диагонализованному виду. Иначе говоря, существуют формальные ряды

$$
T=\sum_{k=0}^{\infty} T(k) \lambda^{-k}, \quad h=\sum_{k=0}^{\infty} h(k) \lambda^{-k}
$$

такие, что оператор $L_{0}:=T^{-1} L T$ является оператором с диагональными коэффициентами, $L_{0}=D_{n}^{-1} h Z$. Это представление позволяет эффективно описать класс полудискретных моделей, для которых $L$ является оператором Лакса. Следуя общему подходу, развитому в работе [8], построим класс $Z_{L}$ формальных рядов вида

$$
A=\sum_{k=-N}^{\infty} A(k) \lambda^{-k}
$$


коммутирующих с оператором $L$. Здесь $A(k)$ - матрица размера $2 \times 2$. Применяя преобразование сопряжения к равенству $[L, A]=0$, находим

$$
\left[L_{0}, A_{0}\right]=0, \quad A_{0}:=T^{-1} A T=\sum_{k=-N}^{\infty} A_{0}(k) \lambda^{-k} .
$$

Лемма 1. Коэфбициенты $A_{0}(k)$ ряда (6.2) суть диагональные матрицъ, не зависящие от $n$.

Схема дОКАзАтЕЛЬСтвА. Нетрудно заметить, что из условия $\left[L_{0}, A_{0}\right]=0$ следует $D_{n}\left(A_{0}\right) h=h Z A_{0} Z^{-1}$. Это уравнение имеет вид $(2.8)$, где потенциал $P(n, \lambda)$ заменен на диагональный ряд $h$. Сравнивая коэффициенты при степенях $\lambda$ в этом уравнении, мы без труда завершаем доказательство леммы.

Обратное утверждение тоже справедливо. Для любого формального ряда

$$
A_{0}=\sum_{k=-N}^{\infty} A_{0}(k) \lambda^{-k}
$$

с диагональными коэффициентами $A_{0}(k)$, не зависящими от $n$, формальный ряд $A=T A_{0} T^{-1}$ лежит в классе $Z_{L}$.

ЗАмЕчаниЕ 1 . Для любого $k$ коэффициент $A(k)$ зависит от конечного числа сдвигов переменной $c$.

В результате мы имеем полное описание множества $Z_{L}$.

Возьмем произвольный ряд $A \in Z_{L}$ и разложим в сумму $A=A_{+}+A_{-}$, где

$$
A_{+}=\sum_{k=-N}^{-1} A(k) \lambda^{-k}+B_{+}, \quad A_{-}=\sum_{k=1}^{\infty} A(k) \lambda^{-k}+B_{-} .
$$

Матрицы $B_{ \pm}$выберем в виде

$$
B_{+}=\left(\begin{array}{cc}
* & * \\
0 & *
\end{array}\right), \quad B_{-}=\left(\begin{array}{cc}
* & 0 \\
* & *
\end{array}\right) .
$$

Здесь и далее звездочками обозначены матричные элементы, вид которых не фиксируется никакими условиями. Остается произвол в выборе диагональных элементов матриц $B_{+}, B_{-}$. Выберем их как функции, зависящие от конечного числа матриц из набора $\{A(k)\}_{k=0}^{\infty}$. Например, можно положить

$$
B_{+}=\left(\begin{array}{cc}
* & * \\
0 & *
\end{array}\right), \quad B_{-}=\left(\begin{array}{cc}
0 & 0 \\
* & 0
\end{array}\right) .
$$

Представление (6.3) гарантирует, что функции $A_{+}$и $Z A_{+} Z^{-1}$ являются полиномами по $\lambda$, в то время как ряды $A_{-}$и $Z A_{-} Z^{-1}$ не содержат членов с положительными степенями $\lambda$. 
ПрЕДЛОЖЕНИЕ 3. Условие совместности системы линейных уравнений

$$
L \phi=\phi, \quad \phi_{t}=A_{+} \phi
$$

эквивалентно дифференииально-разностному уравнению вида

$$
\frac{d c}{d t}=F\left(c, c_{ \pm 1}, c_{ \pm 2}, \ldots, c_{ \pm r}\right)
$$

ДоказАтЕЛьство. Подставим представление $A=A_{+}+A_{-}$в равенство $[L, A]=0$ и найдем

$$
r(\lambda):=c Z A_{+} Z^{-1}-D_{n}\left(A_{+}\right) c=-c Z A_{-} Z^{-1}+D_{n}\left(A_{-}\right) c .
$$

В силу формул (6.3) имеем $r(\lambda) \equiv$ const, поскольку левая часть равенства (6.7) является полиномом по $\lambda$, в то время как правая часть не содержит положительных степеней $\lambda$. Следовательно, полагая $r=d c / d t$, мы получаем самосогласованное равенство. Ясно, что уравнение (6.7) порождает два соотношения

$$
\left[L, \frac{d}{d t}-A_{+}\right]=0, \quad\left[L, \frac{d}{d t}-A_{-}\right]=0,
$$

первое из которых есть не что иное, как условие совместности системы (6.5). Заметим, что согласно замечанию 1 и условию нормировки (6.3) коэффициенты полинома $A_{+}=A_{+}(\lambda)$ являются функциями, зависящими от конечного набора переменных из $c, c_{ \pm 1}, c_{ \pm 2}, \ldots$

Система (6.6) является недоопределенной. Она не меняется при заменах вида $c^{\prime}=D_{n}\left(w^{-1}\right) c w$, где матрица $w$ удовлетворяет условию $[w, Z]=0$. Эта замена переменных соответствует следующему калибровочному преобразованию системы (6.5): $L \rightarrow w^{-1} L w$ и $A \rightarrow w^{-1} A w-w^{-1} w_{t}$. Поэтому на систему нужно наложить некоторые дополнительные ограничения. Условия $c_{11}=$ const, $c_{22}=$ const сводят оператор (6.1) к оператору Лакса иерархии Абловица-Ладика [30] (см. также работу [31]). Это условие согласуется с динамикой, заданной уравнениями (6.6), если нормировка имеет вид (6.4). Действительно, сравнение коэффициентов при $\lambda^{0}$ в уравнении $d c / d t=-c Z A_{-} Z^{-1}+D_{n}\left(A_{-}\right) c$ дает

$$
\frac{d c_{11}}{d t}=\frac{d c_{22}}{d t}=0
$$

Заметим, что имеется еще одна диагонализация оператора (6.1). Действительно, если $c_{22} \neq 0, \operatorname{det} c \neq 0$, то в соответствии со второй частью предложения 1 можно построить формальные ряды по отрицательным степеням $\lambda$,

$$
T^{\prime}=\sum_{k=0}^{\infty} T^{\prime}(k) \lambda^{-k}, \quad h^{\prime}=\sum_{k=0}^{\infty} h^{\prime}(k) \lambda^{-k},
$$

такие, что оператор $L_{0}^{\prime}:=T^{\prime-1} L T^{\prime}$ имеет вид $L_{0}^{\prime}=D_{n}^{-1} h^{\prime} Z$, где коэффициенты $h^{\prime}(k)$ формального ряда $h^{\prime}$ - диагональные матрицы и, кроме того, коэффициенты $T^{\prime}(k)$ ряда $T^{\prime}$ зависят от конечного числа переменных из набора $c, D_{n}^{ \pm 1} c, D_{n}^{ \pm 2} c, \ldots$ Применяя рассуждения, приведенные выше, можно показать, что существует еще одна 
иерархия симметрий. Выберем $c_{11}=c_{22}=1$, положим $c_{21}=u, c_{12}=v$, тогда представители обеих иерархий являются симметриями следующей системы дискретных уравнений:

$$
\begin{aligned}
u_{1,0}-u_{0,1} & =\varepsilon u_{1,1}\left(1-u_{0,1} v_{1,0}\right), \\
v_{0,1}-v_{1,0} & =\varepsilon v\left(1-u_{0,1} v_{1,0}\right) .
\end{aligned}
$$

Эта система допускает пару Лакса вида

$$
L=D_{n}^{-1}\left(\begin{array}{cc}
1 & v \\
u & 1
\end{array}\right) Z, \quad M=D_{m}^{-1}\left(\begin{array}{cc}
\lambda+\varepsilon\left(1-u_{-1,1} v\right) & v \\
\lambda u_{-1,1} & 1
\end{array}\right) .
$$

Можно показать, что в непрерывном пределе система (6.8) переходит в НУШ (см. раздел 7 ниже).

Для иллюстрации метода вычисления симметрий рассмотрим пример.

ПримеР 1. Полагая $c_{11}=1, \operatorname{det} c=1$, сведем сомножитель $c$ в операторе (6.1) к виду (см. формулы (4.2))

$$
c=\left(\begin{array}{cc}
1 & v \\
u & 1+u v
\end{array}\right) .
$$

Для диагонализации оператора воспользуемся предложением 1. Оператор $T$ легко найти, пользуясь формулой (4.3). Выберем затравочный ряд $A_{0}$ в следующем простом виде: $A_{0}=\operatorname{diag}(\lambda / 2,-\lambda / 2)$; его след равен нулю. Построим ряд $A=T^{-1} A_{0} T$, коммутирующий с оператором $L$. Выпишем в явном виде два первых члена ряда $A=A_{-1} \lambda+A_{0}+A_{1} \lambda^{-1}+\cdots$ :

$$
A=\left(\begin{array}{cc}
\frac{1}{2} & 0 \\
u_{-1} & -\frac{1}{2}
\end{array}\right) \lambda+\left(\begin{array}{cc}
-u_{-1} v & v \\
u_{-2}-u_{-1}^{2} v & u_{-1} v
\end{array}\right)+\cdots
$$

Выберем $A_{+}$согласно условию нормировки (6.4):

$$
A_{+}=\left(\begin{array}{cc}
\frac{1}{2} & 0 \\
u_{-1} & -\frac{1}{2}
\end{array}\right) \lambda+\left(\begin{array}{cc}
-u_{-1} v & v \\
0 & u_{-1} v
\end{array}\right) .
$$

Тогда равенство $\left[L, d / d t-A_{+}\right]=0$ приводит к известной системе уравнений [27]

$$
u_{t}=-u_{-1}+u v^{2}, \quad v_{t}=v_{1}-u^{2} v
$$

Завершая этот раздел, приведем еще одну систему уравнений, связанную с дальнейшей редукцией оператора (6.1):

$$
\begin{aligned}
& a_{1,0}\left(1-\kappa u^{2}\right)=a\left(1-\kappa u_{0,1}^{2}\right), \\
& a_{1,0}\left(u-u_{1,1}\right)=u_{0,1}-u_{1,0},
\end{aligned}
$$

где $\kappa= \pm 1$. Эта система является условием коммутирования операторов

$$
L=D_{n}^{-1}\left(\begin{array}{cc}
1 & \kappa u \\
u & 1
\end{array}\right) Z, \quad M=D_{m}^{-1}\left(\begin{array}{cc}
\lambda+a & \kappa\left(u-a u_{0,1}\right) \\
\lambda\left(u-a u_{0,1}\right) & a \lambda+1
\end{array}\right) .
$$


Исключая переменную $а$ из системы (6.9), приходим к уравнению на шеститочечном графе

$$
\frac{\left(u_{0,1}-u_{1,0}\right)\left(1-\kappa u^{2}\right)}{u-u_{1,1}}=\frac{\left(u_{-1,1}-u\right)\left(1-\kappa u_{0,1}^{2}\right)}{u_{-1,0}-u_{0,1}} .
$$

В непрерывном пределе это уравнение переходит в гиперболическое уравнение

$$
\kappa u u_{x}\left(u_{x}^{2}-u_{t}^{2}\right)+2\left(1-\kappa u^{2}\right)\left(u_{t} u_{x t}-u_{x} u_{t t}\right)=0 .
$$

\section{7. ПРИМЕРЫ МАТРИЧНЫХ МОДЕЛЕЙ}

7.1. Дискретный вариант "матричного" НУШ. Система (6.8) допускает простое матричное обобщение

$$
\begin{aligned}
U_{1,0}-U_{0,1} & =\varepsilon U_{1,1}\left(E_{1}-V_{1,0} U_{0,1}\right), \\
V_{0,1}-V_{1,0} & =\varepsilon\left(E_{1}-V_{1,0} U_{0,1}\right) V .
\end{aligned}
$$

Пара Лакса для этой системы записывается с использованием блочных матриц:

$$
L=D_{n}^{-1}\left(\begin{array}{cc}
\lambda E_{1} & V \\
\lambda U & E_{2}
\end{array}\right), \quad M=D_{m}^{-1}\left(\begin{array}{cc}
(\lambda+\varepsilon) E_{1}-\varepsilon V U_{-1,1} & V \\
\lambda U_{-1,1} & E_{2}
\end{array}\right),
$$

где $E_{1}, E_{2}$ - единичные матрицы размера $k \times k$ и $l \times l$, полевые переменные $U$ и $V$ матрицы размера $l \times k$ и $k \times l$ соответственно, а $\varepsilon=\varepsilon(m)$ - заданная функция.

Оператор $L$ в (7.2) записан в требуемой специальной форме, так как он может быть представлен в виде $L=D_{n}^{-1} c Z$. Но он не удовлетворяет условиям предложения 1 , поскольку здесь мы имеем $Z=\operatorname{diag}(\lambda, \ldots, \lambda, 1, \ldots, 1)$, и некоторые из показателей $\gamma_{i}$ совпадают. Однако оператор $L$ может быть “диагонализован” в несколько другом смысле. В этом случае формальные ряды $T, h$ и $S$ являются блочными матрицами:

$$
\begin{aligned}
T(\lambda) & =1+\sum_{k=0}^{\infty}\left(\begin{array}{cc}
0 & T_{12}(k) \\
T_{21}(k) & 0
\end{array}\right) \lambda^{-k}, \\
h(\lambda) & =\sum_{k=0}^{\infty}\left(\begin{array}{cc}
h_{11}(k) & 0 \\
0 & h_{22}(k)
\end{array}\right) \lambda^{-k} \\
S(\lambda) & =\sum_{k=l}^{\infty}\left(\begin{array}{cc}
S_{11}(k) & 0 \\
0 & S_{22}(k)
\end{array}\right) \lambda^{-k}
\end{aligned}
$$

В результате получаем

$$
\begin{aligned}
& T=\left(\begin{array}{cc}
E_{1} & 0 \\
U_{-1,0} & E_{2}
\end{array}\right)+\left(\begin{array}{cc}
0 & -V \\
\left(E_{2}-U_{-1,0} V_{-1,0}\right) U_{-2,0} & 0
\end{array}\right) \lambda^{-1}+\cdots, \\
& h=\left(\begin{array}{cc}
E_{1} & 0 \\
0 & E_{2}-U V
\end{array}\right)+\left(\begin{array}{cc}
V U_{-1,0} & 0 \\
0 & -U V_{1,0}\left(E_{2}-U V\right)
\end{array}\right) \lambda^{-1}+\cdots, \\
& S=\left(\begin{array}{cc}
E_{1} & 0 \\
0 & 0
\end{array}\right) \lambda+\left(\begin{array}{cc}
\varepsilon E_{1}-\varepsilon V_{-1,1} U_{-2,1}+V_{-1,1} U_{-2,1} & 0 \\
0 & E_{2}-U_{-1,1} V
\end{array}\right)+\cdots .
\end{aligned}
$$


Законы сохранения выводятся из уравнений

$$
\left(D_{m}-1\right) \ln \operatorname{det} h_{j j}=\left(D_{n}-1\right) \ln \operatorname{det} S_{j j}, \quad j=1,2 .
$$

Зададим $U$ и $V$ в (7.2) как вектор-столбец и вектор-строку,

$$
U^{\mathrm{T}}=\left(u^{(1)}, u^{(2)}, \ldots, u^{(k)}\right), \quad V=\left(v^{(1)}, v^{(2)}, \ldots, v^{(k)}\right),
$$

тогда, очевидно, произведение $V_{1,0} U_{0,1}=\sum_{j=1}^{k} u_{0,1}^{(j)} v_{1,0}^{(j)}$ является скаляром. В этом случае система (7.1) переписывается следующим образом:

$$
\begin{aligned}
& u_{1,0}^{(i)}-u_{0,1}^{(i)}=\left(1-\sum_{j=1}^{k} u_{0,1}^{(j)} v_{1,0}^{(j)}\right) \varepsilon u_{1,1}^{(i)}, \\
& v_{0,1}^{(i)}-v_{1,0}^{(i)}=\left(1-\sum_{j=1}^{k} u_{0,1}^{(j)} v_{1,0}^{(j)}\right) \varepsilon v^{(i)} .
\end{aligned}
$$

Ее можно интерпретировать как интегрируемую дискретизацию “векторного” НУШ, которое описывает передачу поляризованных импульсов по оптическим волокнам [32]. Формальный непрерывный предел для системы на решетке (7.5) будет найден ниже.

Система (7.5) допускает бесконечную последовательность законов сохранения. Они выводятся из соотношений (7.3). Первые из них имеют вид

$$
\begin{aligned}
& \left(D_{n}-1\right) \ln \left(1-V U_{-1,1}\right)=\left(D_{m}-1\right) \ln (1-V U), \\
& \left(D_{n}-1\right)\left(V_{0,1} U_{-1,1}-\varepsilon V U_{-1,1}\right)=\left(D_{m}-1\right)\left(V_{1,0} U\right) .
\end{aligned}
$$

7.2. Дискретный вариант "векторного" НУШ с производной. Система (4.1) также допускает "векторное" обобщение

$$
\begin{gathered}
U_{0,1}-U_{1,0}+U_{1,1}\left(V_{1,0}\left(U_{0,1}-U_{1,0}\right)+\varepsilon\right)=0, \\
V_{1,0}-V_{0,1}+\left(\left(V_{1,0}-V_{0,1}\right) U_{0,1}+\varepsilon\right) V=0,
\end{gathered}
$$

где $U$ и $V$ - вектор-столбец и вектор-строка, определенные в (7.4). Система (7.6) является условием коммутирования операторов

$$
L=D_{n}^{-1}\left(\begin{array}{cc}
\lambda+V U & V \\
U & E
\end{array}\right), \quad M=D_{m}^{-1}\left(\begin{array}{cc}
(\lambda+\varepsilon)+V U_{-1,1} & V \\
U_{-1,1} & E
\end{array}\right),
$$

где $E$ - единичная матрица размера $(k-1) \times(k-1)$, а $\varepsilon=\varepsilon(m)$ - заданная функция. В координатном представлении система (7.6) принимает вид

$$
\begin{gathered}
u_{0,1}^{(i)}-u_{1,0}^{(i)}+u_{1,1}^{(i)}\left(\sum_{j=1}^{k}\left(v_{1,0}^{(j)} u_{0,1}^{(j)}-v_{1,0}^{(j)} u_{1,0}^{(j)}\right)+\varepsilon\right)=0 \\
v_{1,0}^{(i)}-v_{0,1}^{(i)}+v^{(i)}\left(\sum_{j=1}^{k}\left(v_{1,0}^{(j)} u_{0,1}^{(j)}-v_{0,1}^{(j)} u_{0,1}^{(j)}\right)+\varepsilon\right)=0 .
\end{gathered}
$$

В следующем пункте мы докажем, что система (7.6) представляет собой дискретный вариант "векторного" НУШ с производной. 
7.3. Вычисление непрерывных пределов. Вычислим непрерывные пределы и тем самым покажем, что системы (7.1) и (7.6) являются дискретизациями матричного НУШ и "векторного" НУШ с производной соответственно. Здесь мы положили $\varepsilon(m+1)=\varepsilon(m)$.

Начнем с системы (7.1). Пусть $U(n, m)=r(t, x), V(n, m)=s(t, x), x=m \delta$, $t=\delta^{2} n / 2, \varepsilon=\delta^{2} / 2$. Тогда, очевидно, сдвиги переменных при $\delta \rightarrow 0$ запишутся в виде

$$
\begin{aligned}
U_{-1,0} & =r-\frac{\delta^{2}}{2} r_{t}+o\left(\delta^{2}\right), \\
V_{1,0} & =s+\frac{\delta^{2}}{2} s_{t}+o\left(\delta^{2}\right), \\
U_{0,-1} & =r-\delta r_{x}+\frac{\delta^{2}}{2} r_{x x}+o\left(\delta^{2}\right), \\
V_{0,1} & =s+\delta s_{x}+\frac{\delta^{2}}{2} s_{x x}+o\left(\delta^{2}\right) .
\end{aligned}
$$

Подставим полученные выражения в систему (7.1), переписанную как

$$
\begin{aligned}
U_{0,-1}-U_{-1,0} & =\varepsilon U\left(E_{1}-V_{0,-1} U_{-1,0}\right), \\
V_{0,1}-V_{1,0} & =\varepsilon\left(E_{1}-V_{1,0} U_{0,1}\right) V .
\end{aligned}
$$

После некоторых упрощений получаем при $\delta \rightarrow 0$

$$
\begin{aligned}
r_{t}-\frac{2}{\delta} r_{x}+r_{x x} & =r\left(E_{1}-s r\right)+o(\delta), \\
-s_{t}+\frac{2}{\delta} s_{x}+s_{x x} & =\left(E_{1}-s r\right) s+o(\delta) .
\end{aligned}
$$

Введем новые переменные $x=x^{\prime}-\delta t^{\prime} / 2, t=t^{\prime}, r=e^{t} R, s=e^{-t^{\prime}} S$ и, учитывая, что

$$
\frac{\partial}{\partial t^{\prime}}=\frac{\partial}{\partial t}-\frac{2}{\delta} \frac{\partial}{\partial x}, \quad \frac{\partial}{\partial x^{\prime}}=\frac{\partial}{\partial x},
$$

получим при $\delta \rightarrow 0$ матричное НУШ

$$
R_{t^{\prime}}+R_{x^{\prime} x^{\prime}}+R S R=0, \quad-S_{t^{\prime}}+S_{x^{\prime} x^{\prime}}+S R S=0 .
$$

Покажем, что система (7.6) является дискретным вариантом "векторного" НУШ с производной. Вычислим непрерывный предел системы (7.6), введя обозначения $U(n, m)=\delta r(x, t), V(n, m)=\delta s(x, t), x=\delta^{2} m, t=\delta^{4} n / 2, \varepsilon=\delta^{5}$. Разложение Тейлора для сдвигов переменных при $\delta \rightarrow 0$ имеет вид

$$
\begin{aligned}
U_{-1,0} & =\delta r-\frac{\delta^{5}}{2} r_{t}+o\left(\delta^{5}\right), \\
V_{1,0} & =\delta s+\frac{\delta^{5}}{2} s_{t}+o\left(\delta^{5}\right), \\
U_{0,-1} & =\delta r-\delta^{3} r_{x}+\frac{\delta^{5}}{2} r_{x x}+o\left(\delta^{5}\right), \\
V_{0,1} & =\delta s+\delta^{3} s_{x}+\frac{\delta^{5}}{2} s_{x x}+o\left(\delta^{5}\right) .
\end{aligned}
$$


Затем подставим найденные значения в систему (7.6), переписанную как

$$
\begin{array}{r}
U_{-1,0}-U_{0,-1}+U\left(V_{0,-1}\left(U_{-1,0}-U_{0,-1}\right)+\varepsilon\right)=0 \\
V_{1,0}-V_{0,1}+\left(\left(V_{1,0}-V_{0,1}\right) U_{0,1}+\varepsilon\right) V=0 .
\end{array}
$$

В результате получаем

$$
\begin{aligned}
-\frac{\delta^{5}}{2} r_{t}+\delta^{3} r_{x}-\frac{\delta^{5}}{2} r_{x x}-\delta^{5} r s r_{x} & =o\left(\delta^{5}\right), \\
\frac{\delta^{5}}{2} s_{t}-\delta^{3} s_{x}-\frac{\delta^{5}}{2} s_{x x}-\delta^{5} s_{x} r s & =o\left(\delta^{5}\right) .
\end{aligned}
$$

Введем новые независимые переменные $t^{\prime}, x^{\prime}$ такие, что $x=x^{\prime}-\delta^{2} t^{\prime} / 2, t=t^{\prime}$ и, учитывая соотношения

$$
\frac{\partial}{\partial x^{\prime}}=\frac{\partial}{\partial x}, \quad \frac{\partial}{\partial t^{\prime}}=\frac{\partial}{\partial t}-\frac{2}{\delta^{2}} \frac{\partial}{\partial x},
$$

вычислим предел при $\delta \rightarrow 0$. Тогда система (7.7) дает нам "векторную" версию НУШ с производной

$$
r_{t^{\prime}}+\frac{1}{2} r_{x^{\prime} x^{\prime}}+r s r_{x^{\prime}}=0, \quad-s_{t^{\prime}}+\frac{1}{2} s_{x^{\prime} x^{\prime}}+s_{x^{\prime}} r s=0 .
$$

\section{8. ЗАКЛЮЧЕНИЕ}

Метод формальной диагонализации пары Лакса, предложенный в работе [8], является эффективным средством построения законов сохранения и высших симметрий нелинейных интегрируемых уравнений в частных производных. В настоящей работе метод диагонализации пары Лакса обобщается на дискретный случай. Эффективность такого подхода иллюстрируется содержательными примерами, включающими дискретное потенциированное уравнение Кортевега-де Фриза, иерархию Абловица-Ладика, одевающие цепочки Леви-Ямилова и Веселова-Шабата-Ямилова, цепочку Тоды с дискретным временем, соответствующую алгебре Ли $A_{1}^{(1)}$. Представлены новые примеры интегрируемых дискретных уравнений и систем.

Настоящая статья стимулирована докладом [13] и имеет с ним некоторые пересечения, но изложение ведется независимо.

Благодарности. Наша работа выполнена при частичной финансовой поддержке РФФИ (гранты № 11-01-97005-р-поволжье_а, 12-01-31208-мол_а, 13-01-00070_а) и Федеральной целевой программы "Научные и педагогические кадры инновационной России на 2009-2013 гг.” (контракт № 2012-1.5-12-000-1003-011).

\section{Список литературы}

[1] Э. А. Коддингтон, Н. Левинсон, Теория обыкновенных дифференциальных уравнений, ЛКИ, М., 2010.

[2] В. Вазов, Асимптотические разложения решений обыкновенных дифференииальных уравнений, Мир, М., 1968.

[3] М. Абловиц, Х. Сигур, Солитонъ и метод обратной задачи, Мир, М., 1987. 
[4] В. Е. Захаров, С. В. Манаков, С.П. Новиков, Л. П. Питаевский, Теория солитонов: метод обратной задачи, Наука, М., 1980.

[5] Л. А. Тахтаджян, Л. Д. Фаддеев, Гамильтонов подход в теории солитонов, Наука, М., 1986.

[6] В. Е. Захаров, А. Б. Шабат, Функи. анализ и его прил., 8:3 (1974), 43-53.

[7] В. Е. Захаров, А. Б. Шабат, Функи. анализ и его прил., 13:3 (1979), 13-22.

[8] В. Г. Дринфельд, В. В. Соколов, “Алгебры Ли и уравнения типа Кортевега-де Фриза”, Итоги науки и техн. Сер. Соврем. пробл. мат. Нов. достиж., 24, ВИНИТИ, М., 1984, $81-180$.

[9] V. Caudrelier, Internat. J. Geom. Methods Mod. Phys., 5:7 (2008), 1085-1108.

[10] I. Habibullin, A. Kundu, Nucl. Phys. B, 795:3 (2008), 549-568, arXiv: 0709.4611.

[11] D. J. Zhang, J. W. Cheng, Y. Y. Sun, Deriving conservation laws for ABS lattice equations from Lax pairs, arXiv: 1210.3454 .

[12] J. W. Cheng, D. J. Zhang, Conservation laws of some lattice equations, arXiv: 1212.2319.

[13] A. V. Mikhailov, "Formal diagonalisation of Darboux transformation and conservation laws of integrable PDEs, PD $\Delta \mathrm{Es}$, and P $\Delta \mathrm{Es}$ ", Доклад на Международном научном семинаре "Геометрические структуры в интегрируемых системах" (Москва, МГУ, 30 октября-2 ноября 2012), http://www.mathnet.ru/php/presentation.phtml?option_lang= eng\&presentid $=5934$.

[14] R. N. Garifullin, A. V. Mikhailov, R. I. Yamilov, A quad graph equation with nonstandard generalized symmetry structure (in preparation).

[15] И. Т. Хабибуллин, Зап. научн. сем. ЛОМИ, 146 (1985), 137-146.

[16] А. Б. Шабат, "Нелинейные уравнения и задача Римана", Труды Всесоюзной конференции по уравнениям с частными производными, посвященная 75-летию со дня рождения академика И. Г. Петровского, Изд-во Московск. ун-та, М., 1978, 242-245.

[17] Ф.Р. Гантмахер, Теория матрии, Физматлит, М., 2004.

[18] А. В. Михайлов, А. Б. Шабат, Р. И. Ямилов, УМН, 42:4(256) (1987), 3-53.

[19] A. V. Mikhailov, "From automorphic Lie algebras to discrete integrable systems", Seminar talk in "Discrete Integrable Systems" (Isaac Newton Institute for Mathematical Sciences, Cambridge, 17 June 2009), http://www.newton.ac.uk/programmes/DIS/seminars/ 2009061714001.html.

[20] R. N. Garifullin, I. T. Habibullin, M. V. Yangubaeva, SIGMA, 8 (2012), 062, 33 pp.

[21] H. D. Wahlquist, F. B. Estabrook, Phys. Rev. Lett., 31:23 (1973), 1386-1390.

[22] A. G. Rasin, J. Phys. A, 43:23, 235201, 12 pp., arXiv: 1001.0724.

[23] А. В. Михайлов, Дж. П. Ванг, П. Ксенитидис, ТМФ, 167:1 (2011), 23-49.

[24] F. W. Nijhoff, G. R. W. Quispel, H. W. Capel, Phys. Lett. A, 97:4 (1983), 125-128.

[25] D. Levi, R. I. Yamilov, J. Phys. A, 42:45 (2009), 454012, 18 pp., arXiv: 0902.4421.

[26] M. Toda, Proc. Theor. Phys. Suppl., 45 (1970), 174-200.

[27] А. Б. Шабат, Р. И. Ямилов, Алгебра и анализ, 2:2 (1990), 183-208.

[28] A. Shabat, Inverse Problems, 8:2 (1992), 303-308.

[29] А. П. Веселов, А. Б. Шабат, Функи. анализ и его прил., 27:2 (1993), 1-21.

[30] M. J. Ablowitz, M. J. Ladik, J. Math. Phys., 16:3 (1975), 598-603.

[31] F. Pempinelli, M. Boiti, J. Leon, "Bäcklund and Darboux transformation for the Ablowitz-Ladik spectral problem", Nonlinear Physics: Theory and Experiment (Lecce, Italy, June 29 - July 7, 1995), eds. E. Alfinito, M. Boiti, L. Martina, F. Pempinelli, World Sci. Publ, Singapore, 1996, 261-268.

[32] С. В. Манаков, ЖЭТФ, 65:10 (1973), 505-516. 